\title{
Characteristic Attributes of Multiple Cascaded Terahertz Metasurfaces with Magnetically Tunable Subwavelength Resonators
}

\author{
Andriy E. Serebryannikov,* Akhlesh Lakhtakia, and Ekmel Ozbay
}

The characteristics of multiple cascaded metasurfaces comprising $\mathrm{H}$-shaped, magnetostatically controllable, subwavelength terahertz $(\mathrm{THz})$ resonators made of InAs were systematically investigated, using a commercial solver based on the finite-integration method, for the design of tunable filters. Three configurations of the biasing magnetostatic field were compared with each other as well as with the bias-free configuration for filtering of normally incident linearly polarized plane waves. A close study of only one metasurface was found sufficient to broadly determine the sensitivity to the direction of the magnetostatic field and the bandwidth of a stopband. Furthermore, the effects of metasurface geometry and biasing field can be considered separately for initial design purposes. All features in the transmittance spectra for the bias-free configuration that are related to the number of cascaded metasurfaces are also observed when the biasing magnetostatic field is applied. The coupling of adjacent metasurfaces in a cascade is strongly affected by the relative permittivity and the thickness of the spacer between the two metasurfaces. The spectral locations of stopbands scale with respect to the spacer's relative permittivity, the scaling rule being different from a classical one. The stopbands are redshifted when the spacer thickness is increased, with the redshift dependent on the polarization of the incident plane wave. Inter-metasurface coupling and inter-resonator coupling on the same metasurface affect the spectral location of a stopband in opposite ways. On-off type switching can be obtained by changing the orientation of magnetostatic field. The elucidated characteristics are expected to be important for not only filters but also other tunable $\mathrm{THz}$ devices.

\section{Introduction}

The unprecedented ability to control the frequency, polarization state, and the direction of propagation of electromagnetic plane waves and beams offered by metasurfaces has made these planar analogs of metamaterials very attractive subjects for both theoretical and experimental research. ${ }^{[1-3]}$ Generally, a metasurface is a periodic or a nonperiodic array of subwavelength elements, whether resonant or non-resonant, typically placed on a dielectric substrate. ${ }^{[3-7]}$ Many interesting effects and useful applications can be obtained by cascading several metasurfaces. ${ }^{[8-13]}$ Furthermore, the coupling of two parallel metasurfaces, whether identical or different, may yield significant effects for reflection, transmission, and polarization conversion. ${ }^{[10,14-16]}$ A study of the cascade of two metasurfaces in which one is the complement of the other in accordance with the Babinet principle must be noted, as it gives a detailed physical insight on the coupling and transmission mechanism. ${ }^{[17]}$

Dynamic control of a metasurface may enable either fine tuning in a wide spectral regime or on-off switching, without mechanical modification of the structure. As the dynamic-control approaches being explored for metasurfaces are, generally speaking, the same as those used earlier for other types of structures and devices, ${ }^{[18-20]}$ relevant experience can be transferred to metasurfaces. The approaches involve the exploitation of thermal, ${ }^{[21-23]}$ magnetostatic, ${ }^{[10,24]}$ electric-field/voltage, ${ }^{[25-28]}$ photoconductive, ${ }^{[29,30]}$ Raman $^{[31]}$ and gas-filling ${ }^{[32]}$ mechanisms, whether in the substrate or the subwavelength elements of a metasurface.

The dynamic-control approach based on the sensitivity of constitutive parameters such as the relative permittivity tensor to variations in the magnitude $B_{0}$ of a magnetostatic field $\mathbf{B}_{0}$ is almost universal. It can be implemented in a very wide frequency range, e.g., from the microwave to the mid-infrared frequencies, provided that materials sensitive to magnetostatic-field variations are available and fulfill specific requirements connected with a concrete application. Therefore, it has been investigated for

DOI: 10.1002/andp.201700252 
metasurfaces as well. ${ }^{[10,33]}$ Undoubtedly, it is most important to realize efficient tunability when the magnetostatic field is relatively weak in magnitude (i.e., when $B_{0}$ does not exceed $1 \mathrm{~T}$ ), so that it cannot affect neighboring equipment.

The simplest way to implement dynamic tunability is the use of a substrate with constitutive parameters that depend on the control mechanism. ${ }^{[29,30]}$ Alternatively, the resonant or nonresonant elements of the metasurface can be made of a dynamically tunable material. ${ }^{[10,22,24,33]}$ The latter approach is desirable because the volume of the elements is much smaller than of the underlying substrate, the implementation of the control mechanism in a smaller region requiring less of the tunable material. Finally, just some parts of these elements can be made of the tunable material. ${ }^{[34-36]}$

In this paper, we elucidate the characteristic attributes of multiple cascaded metasurfaces with magnetically tunable resonators as the subwavelength elements to operate in the terahertz $(\mathrm{THz})$ spectral regime. For definiteness, the resonators of thickness $t$ are $\mathrm{H}$-shaped ${ }^{[10,37,38]}$ and arranged on a square lattice of side $a$ on every metasurface. An $\mathrm{H}$-shaped resonator allows us to distinguish between two Voigt configurations of the applied magnetostatic field, in contrast to Maltese-cross-shaped resonators.

The resonators are made of InAs, a material whose dielectric properties are strongly sensitive in the $\mathrm{THz}$ regime to variations in the strength and the direction of a magnetostatic field. ${ }^{[10,24]}$ With THz filters being the focus, the geometry of the chosen resonators enables high sensitivity to changes in the polarization state and the direction of the incident $\mathrm{THz}$ wave. Although our investigation is focused on filtering applications, the elucidated characteristics are expected to be important for diverse tunable THz devices.

We demonstrate that the coupling of adjacent metasurfaces in a cascade is strongly affected by the relative permittivity $\varepsilon_{d}$ and the thickness $b$ of the substrates, each substrate serving as a spacer between two planar arrays of magnetically tunable subwavelength resonators. The spectral locations of subwavelength resonances are modified by the substrate/spacer's relative permittivity, leading to a scaling rule which differs from a classical one. ${ }^{[39]}$

With all metasurfaces oriented parallel to the plane $z=0$ of a Cartesian coordinate system and considering only $\mathrm{THz}$ waves that are incident normally on the multiple cascaded metasurfaces, we compare three configurations that are distinguished by the orientation of the magnetostatic field:

i. the Faraday configuration with the magnetostatic field aligned parallel to the $z$ axis (i.e., perpendicular to the metasurface planes),

ii. the Voigt-Y configuration in which the magnetostatic field is aligned parallel to the $y$ axis (i.e., parallel to one of the two sides of the unit cell in the metasurfaces), and

iii. the Voigt-X configuration in which the magnetostatic field is aligned parallel to the $x$ axis (i.e., parallel to the other side of the unit cell in the metasurfaces).

For economical presentation of the results, we assume that $B_{0}=$ $1 \mathrm{~T}$ for all three configurations, and we compare the numerical results with those for the bias-free configuration (i.e., $B_{0}=0$ ). We also discuss to some extent the effect of the lattice parameter a. All numerical results presented here were obtained by using CST Microwave Studio, a commercial solver based on the finiteintegration method..$^{[0]}$

The paper is organized as follows. In Section 2, we introduce the geometry of the multiple cascaded metasurfaces as well as the magnetostatically dependent relative permittivity tensor of InAs. Section 3 is dedicated to the identification of the effects of intermetasurface coupling as well as of the coupling of resonators on the same metasurface. In Section 4, the effects of the relative permittivity $\varepsilon_{d}$ and the thickness $b$ of the substrate/spacer on the transmission and reflection characteristics are presented, and the scaling of subwavelength resonances with appropriate choice of $\varepsilon_{d}$ is considered in detail. Section 5 presents key results for cascades of four and eight metasurfaces. Concluding remarks are provided in Section 6.

\section{Geometry and Constitutive Parameters}

We consider a stack of $M \geq 1$ metasurfaces. When $M>1$, the metasurfaces are identical and stacked as closely as possible, without change of orientation of the resonators on adjacent metasurfaces and with the substrates serving as spacers, as shown in Figure 1. Every metasurface comprises H-shaped subwavelength resonators on a square lattice imprinted on a dielectric substrate.

The lattice parameter $a$ was taken to either equal or exceed $a_{0}=15.56 \mu \mathrm{m}$ for all results reported here. Each resonator contains two $w \times h$ sections and one $w \times l$ section, all of thickness $t=0.5 \mu \mathrm{m}$, the side of dimension $l=9 \mu \mathrm{m}$ being oriented along the $x$ axis, the side of dimension $h=14 \mu \mathrm{m}$ oriented along the $y$ axis, and $w=2.5 \mu \mathrm{m}$. The twofold symmetry of the H-shaped resonators in the $x y$ plane is the reason for the strong sensitivity to the polarization state (either $\mathbf{E}_{\text {inc }} \| \hat{\mathbf{x}}$ or $\mathbf{E}_{\text {inc }} \| \hat{\mathbf{y}}$ ) of the normally incident plane wave. The structure's total thickness is $D=b+t$ when $M=1$ and $D=(M-1) b+M t$ when $M>1$.

In the absence of an external magnetostatic field $\left(B_{0} \equiv 0\right)$, InAs is an isotropic material. Its relative permittivity tensor $\overline{\bar{\varepsilon}}$ then simplifies to a scalar, with the diagonal components specified in the $\mathrm{THz}$ regime all equal as ${ }^{[10,24]}$

$\varepsilon_{x x}=\varepsilon_{y y}=\varepsilon_{z z}=\varepsilon_{\infty}-\frac{\omega_{p}^{2}}{\omega^{2}+i \omega \gamma}$,

and the off-diagonal elements all being zero. Here and hereafter, $\varepsilon_{\infty}=16.3$ is the high-frequency relative permittivity scalar, $\gamma /(2 \pi)=7.5 \times 10^{11} \mathrm{~Hz}$ is the damping parameter, and $\omega_{p}=\sqrt{N e^{2} / \varepsilon_{0} m^{*}}$ is the plasma frequency. With $N=1.04 \times 10^{23} \mathrm{~m}^{-3}$ as the free-career density at room temperature, $m^{*}=0.004 m_{e}$ as the effective career mass, $m_{e}=$ $9.1 \times 10^{-31} \mathrm{~kg}, e=1.6 \times 10^{-19} \mathrm{C}$, and $\varepsilon_{0}=8.854 \times 10^{-12} \mathrm{~F} \mathrm{~m}^{-1}$, we get $\omega_{p}=2.875 \times 10^{14} \mathrm{rad} \mathrm{s}^{-1}$. Thus, InAs is plasmonic $\left[\operatorname{Re}\left(\varepsilon_{x x}\right)<0\right]$ at frequencies lower than $11.3054 \mathrm{THz}$ and nonplasmonic $\left[\operatorname{Re}\left(\varepsilon_{x x}\right)>0\right]$ at frequencies higher than $11.3054 \mathrm{THz}$, but is dissipative $\left[\operatorname{Im}\left(\varepsilon_{x x}\right)>0\right]$ at all frequencies, provided that $B_{0} \equiv 0$. 


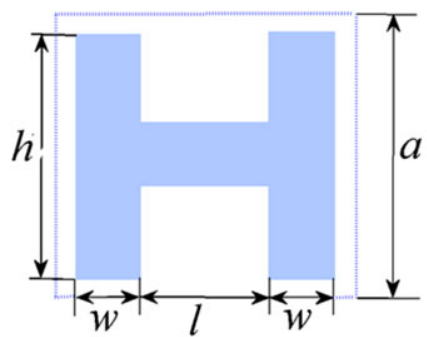

(a)

(d)

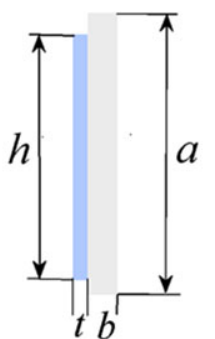

(b)

(e)

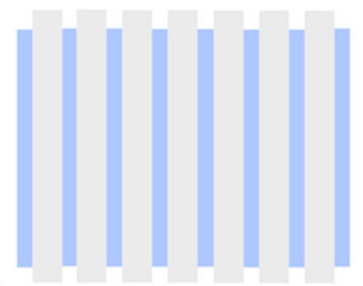

(c)

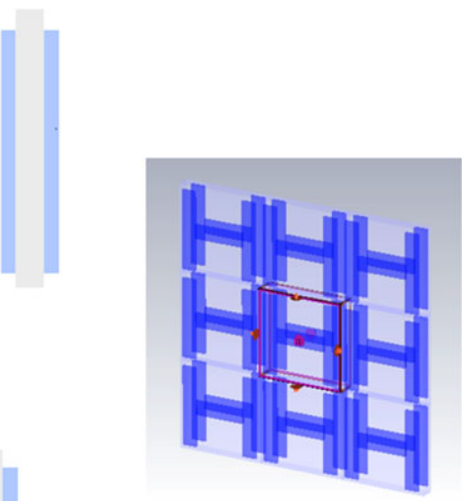

(f)

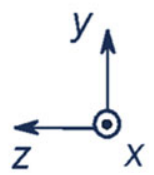

Figure 1. a) Front view of the unit cell of the metasurfaces; side views of the metasurfaces when b) $M=1, c) M=2, d) M=4$, and e) $M=8$; and $\mathrm{f})$ perspective view $(3 \times 3$ unit cells) when $M=2$, the spacer is shown as completely transparent, and the $\mathrm{H}$-shaped resonators are shown as semi-transparent. The axes of the Cartesian coordinate system shown are related to b)-e).

In the Faraday configuration, $\mathbf{B}_{0}=B_{0} \hat{\mathbf{z}}$ and the non-zero components of $\overline{\bar{\varepsilon}}$ are

$\varepsilon_{x x}=\varepsilon_{\gamma Y}=\varepsilon_{\infty}-\frac{\omega_{p}^{2}\left(\omega^{2}+i \gamma \omega\right)}{\left(\omega^{2}+i \gamma \omega\right)^{2}-\omega^{2} \omega_{c}^{2}}$,

$\varepsilon_{x y}=-\varepsilon_{\gamma x}=i \frac{\omega \omega_{c} \omega_{p}^{2}}{\left(\omega^{2}+i \gamma \omega\right)^{2}-\omega^{2} \omega_{c}^{2}}$,

$\varepsilon_{z z}=\varepsilon_{\infty}-\frac{\omega_{p}^{2}}{\omega^{2}+i \omega \gamma}$

where $\omega_{c}=e B_{0} / m^{*}$ is the cyclotron frequency. Simple analysis of Eq. (2) shows that $\varepsilon_{x x}$ and $\varepsilon_{\gamma \gamma}$ may take large values for their real and imaginary parts, much larger than $\varepsilon_{\infty}$. Furthermore, the off-diagonal components of $\overline{\bar{\varepsilon}}$ in Eq. (3) may also possess large magnitudes. Figures 2 and 3 present values of the diagonal and off-diagonal components of $\overline{\bar{\varepsilon}}$ when $B_{0}=0$ and $1 \mathrm{~T}$. One can see that the non-zero components of $\overline{\bar{\varepsilon}}$ of InAs are of high magnitudes in the entire 1-5 THz frequency range when $B_{0}=1 \mathrm{~T}$.

In the Voigt-Y configuration $\left(\mathbf{B}_{0}=B_{0} \hat{\mathbf{y}}\right), \varepsilon_{Y Y}$ is given by the right side of Eq. (4), while $\varepsilon_{x x}=\varepsilon_{z z}$ and $\varepsilon_{z x}=-\varepsilon_{x z}$ are given by the right sides of Eqs. (2) and (3), respectively. Finally, in the Voigt$\mathrm{X}$ configuration $\left(\mathbf{B}_{0}=B_{0} \hat{\mathbf{x}}\right), \varepsilon_{x x}$ is given by the right side of Eq. (4), whereas $\varepsilon_{y Y}=\varepsilon_{z z}$ and $\varepsilon_{z y}=-\varepsilon_{y z}$ are given by the right sides of Eqs. (2) and (3), respectively.

The specularly transmitted electric field is denoted by $\mathbf{E}_{\mathrm{tr}}^{\mathrm{spec}}=$ $\left(\tau_{x x} \hat{\mathbf{x}}+\tau_{\gamma x} \hat{\mathbf{y}}\right) \exp \left(i 2 \pi z / \lambda_{0}\right)$ when $\mathbf{E}_{\text {inc }}=\hat{\mathbf{x}} \exp \left(i 2 \pi z / \lambda_{0}\right)$ and by $\mathbf{E}_{\mathrm{tr}}^{\text {spec }}=\left(\tau_{x y} \hat{\mathbf{x}}+\tau_{y y} \hat{\mathbf{y}}\right) \exp \left(i 2 \pi z / \lambda_{0}\right)$ when $\mathbf{E}_{\text {inc }}=\hat{\mathbf{y}} \exp \left(i 2 \pi z / \lambda_{0}\right)$, where $\lambda_{0}$ is the free-space wavenumber. We computed the spectra of the four specular transmittances $t_{x x}=\left|\tau_{x x}\right|^{2}, t_{\gamma \gamma}=\left|\tau_{\gamma \gamma}\right|^{2}$, $t_{x y}=\left|\tau_{x y}\right|^{2}$, and $t_{\gamma x}=\left|\tau_{\gamma x}\right|^{2}$ for all four configurations of the magnetostatic field (i.e., Faraday, Voigt-Y, Voigt-X, and bias-free). We use $t_{x x}^{(F)}, t_{x x}^{(V Y)}, t_{x x}^{(V X)}$, and $t_{x x}^{(0)}$ to denote the value of $t_{x x}$ in the Faraday, Voigt-Y, Voigt-X, and bias-free configurations; and similarly for the other three specular transmittances.

\section{Coupling of Two Metasurfaces}

Let us begin by demonstrating how the coupling of two metasurfaces can affect overall transmission and why cascading can be desirable, and thereby introduce five design principles. To this end, we compare the transmittance spectra of a single metasurface $(M=1)$ and two coupled metasurfaces $(M=2)$ with $\varepsilon_{d}=1.0$ (i.e., the substrate is absent for $M=1$ and the spacer is vacuous for $M=2$ ). Hence, the transmittance spectra are fully determined by (i) the subwavelength resonances of the individual $\mathrm{H}$-shaped resonators, (ii) the intralayer coupling of a unit cell with other unit cells on the same metasurface, and (iii) the interlayer coupling of two metasurfaces. Couplings of both types can play positive roles for enhancing performance. ${ }^{[17]}$

Figure 4 presents the co-polarized transmittance spectra for the Faraday, Voigt-Y, Voigt-X, and bias-free configurations, for both $\mathbf{E}_{\text {inc }} \| \hat{\mathbf{x}}$ and $\mathbf{E}_{\text {inc }} \| \hat{\mathbf{y}}$. The distance between the metasurfaces for $M=2$ is taken as $b=1 \mu \mathrm{m}$. A clear stopband centered at a frequency $f_{c}$ somewhere between $1.5 \mathrm{THz}$ and $2.5 \mathrm{THz}$ and another between $3.5 \mathrm{THz}$ and $5 \mathrm{THz}$ are evident for all four configurations. The analogous spectra (not shown) for $b=0.75 \mu \mathrm{m}$ have the same features.

Sensitivities to the orientation of $\mathbf{B}_{0}$ and the polarization state of the incident plane wave are evident in Figure 4. Whereas $t_{y Y}^{(0)} \approx t_{y Y}^{(V Y)}$ and $t_{x x}^{(0)} \approx t_{x x}^{(V X)}$, we notice that $t_{y y}^{(V Y)}$ and $t_{y Y}^{(V X)}$ are different from each other, and $t_{x x}^{(V Y)}$ and $t_{x x}^{(V X)}$ are also different from each other, regardless of the value of $M$. Both of the sensitivities had been observed earlier for $\varepsilon_{d}=2.1 .^{[10]}$ 

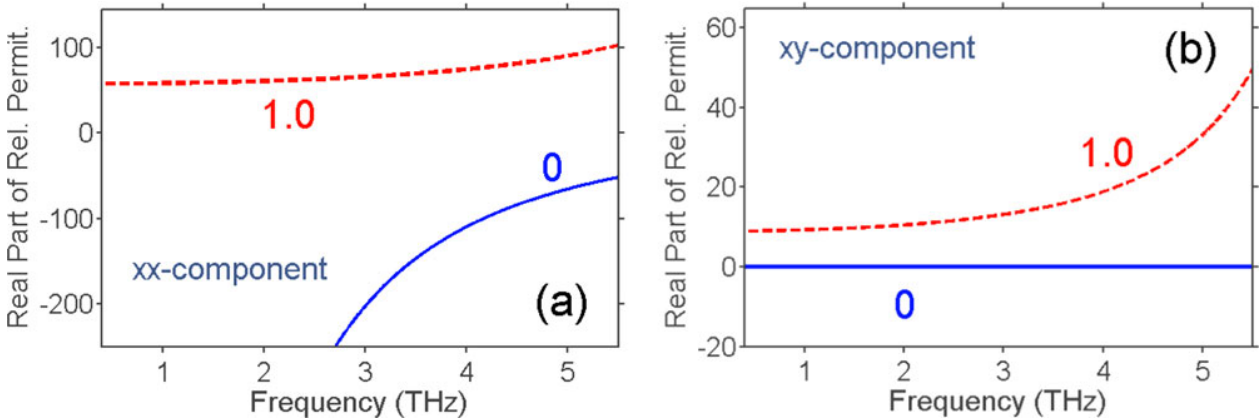

Figure 2. Plots of a) $\operatorname{Re}\left(\varepsilon_{x x}\right)$ and b) $\operatorname{Re}\left(\varepsilon_{x y}\right)$ vs frequency $f \in[0.1,5.5] \mathrm{THz}$ for the Faraday configuration. Note that $\varepsilon_{z z}$ defined in Eq. (4) for any value of $B_{0}$ is the same as $\varepsilon_{x x}$ defined in Eq. (2) for $B_{0}=0$. The values of $B_{0}$ in Tesla are identified.
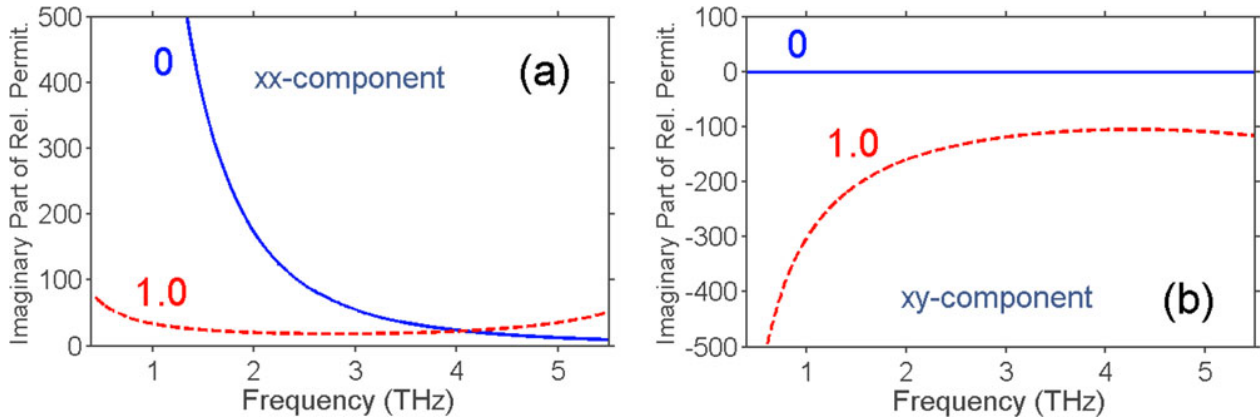

Figure 3. Plots of a) $\operatorname{Im}\left(\varepsilon_{x x}\right)$ and b) $\operatorname{Im}\left(\varepsilon_{x y}\right)$ vs frequency $f \in[0.1,5.5] \mathrm{THz}$ for the Faraday configuration. Note that $\varepsilon_{z z}$ defined in Eq. (4) for any value of $B_{0}$ is the same as $\varepsilon_{x x}$ defined in Eq. (2) for $B_{0}=0$. The values of $B_{0}$ in Tesla are identified.

Furthermore, the stopband is deeper for $M=2$ than for $M=1$ in Figure 4, which characteristic had also been observed earlier for $\varepsilon_{d}=2.1 .^{[10]}$ Clearly from the data presented in Figure 4, both (i) the sensitivities to the orientation of $\mathbf{B}_{0}$ and the polarization state of the incident plane wave as well as (ii) the stopband's depth are mainly governed by the characteristics of a single metasurface and can be modified by cascading two metasurfaces. These weak effects point out the first design principle that a close study of only one metasurface is sufficient to broadly determine the sensitivity to the direction of the magnetostatic field and the bandwidth of the stopband.

The spectra of $t_{x x}^{(F)}$ and $t_{Y y}^{(F)}$ for $M=2$ and $b \in\{1,2.5,5.5\} \mu \mathrm{m}$ are provided in Figure 5, the Faraday configuration being the most sensitive to the magnitude $B_{0}$. A general trend in this figure is the blueshift of $f_{c}$ for decreasing $b$ when $M=2$. This is due to the capacitative coupling between two metasurfaces placed in the proximity of each other. The center frequency $f_{c}$ is redshifted toward that of a single metasurface when $b$ is increased: the capacitative effect weakens, and so does the coupling between the two metasurfaces, with increasing $b$.

The effect of $b$ is clearly different for different polarization states of the incident plane wave, but a quantitative understanding of that effect turned out to be elusive. Every co-polarizedtransmittance spectrum in Figures $4(\mathrm{a}, \mathrm{c})$ and 5(a) actually contains a highly prominent stopband and a much muted stopband, the prominent first stopband occurring with a lower center frequency than the second stopband. Tables 1 and 2 present the center frequencies of the first and second stopbands, respectively, for all configurations, when $M=2$ and $b \in\{1,2.5,5.5\} \mu \mathrm{m}$. For comparison, the results are presented also for $M=1$. Clearly, the strongest redshifts of both the first and the second stopbands by the application of the magnetostatic field occur for the Faraday configuration. Correspondingly, either $t_{x x}$ or $t_{y y}$ is weakly changed in a Voigt configuration when a magnetostatic field of magnitude $1 \mathrm{~T}$ is applied, depending on whether the sign of $\operatorname{Re}\left\{\mathbf{E}_{\text {inc }} \bullet \overline{\bar{\varepsilon}} \bullet \mathbf{E}_{\text {inc }}\right\}$ is preserved under biasing in the direction parallel to $\mathbf{E}_{\text {inc }}$.

The range $\Delta f_{c}^{(b)}$ of the variation of $f_{c}$ which is achievable with $M=2$ by varying $b$ from $1.0 \mu \mathrm{m}$ to $5.5 \mu \mathrm{m}$ can depend on the choice of configuration. For the Faraday configuration, we obtain $\Delta f_{c}^{(b)} \approx 0.2 \mathrm{THz}$ and $0.34 \mathrm{THz}$ for $\mathbf{E}_{\text {inc }} \| \hat{\mathbf{x}}$ and $\mathbf{E}_{\text {inc }} \| \hat{\mathbf{y}}$, respectively; for the Voigt-Y configuration, $\Delta f_{c}^{(b)} \approx 0.2 \mathrm{THz}$ and $0.39 \mathrm{THz}$; for the Voigt-X configuration, $\Delta f_{c}^{(b)} \approx 0.18 \mathrm{THz}$ and $0.31 \mathrm{THz}$; and for the bias-free configuration, $\Delta f_{c}^{(b)} \approx 0.19 \mathrm{THz}$ and $0.44 \mathrm{THz}$. The analogous data for $M=1$ are as follows: 0.3 $\mathrm{THz}$ and $0.45 \mathrm{THz}$ for the Faraday configuration, $0.28 \mathrm{THz}$ and $0.56 \mathrm{THz}$ for the Voigt-Y configuration, $0.31 \mathrm{THz}$ and $0.48 \mathrm{THz}$ for the Voigt-X configuration, and $0.31 \mathrm{THz}$ and $0.56 \mathrm{THz}$ for the bias-free configuration. Thus, variations in $b$ have weaker effects on the range $\Delta f_{c}^{(b)}$ for $\mathbf{E}_{\text {inc }} \| \hat{\mathbf{x}}$ than for $\mathbf{E}_{\text {inc }} \| \hat{\mathbf{y}}$. This is the second design principle identified by us.

As mentioned earlier in this section, coupling between two metasurfaces $(M=2)$ is stronger for smaller $b$. Thus, the case of $M=1$ can be regarded as the limiting case for that of $M=$ 2 as $b$ is increased. This feature qualitatively coincides with the results for coupling of subwavelength (split-ring) resonators obtained earlier by using the Lagrange formalism. ${ }^{[41-44]}$ However, the limiting case must be obtained with relatively small values of $b$, because the underlying physics presents a richer palette of 

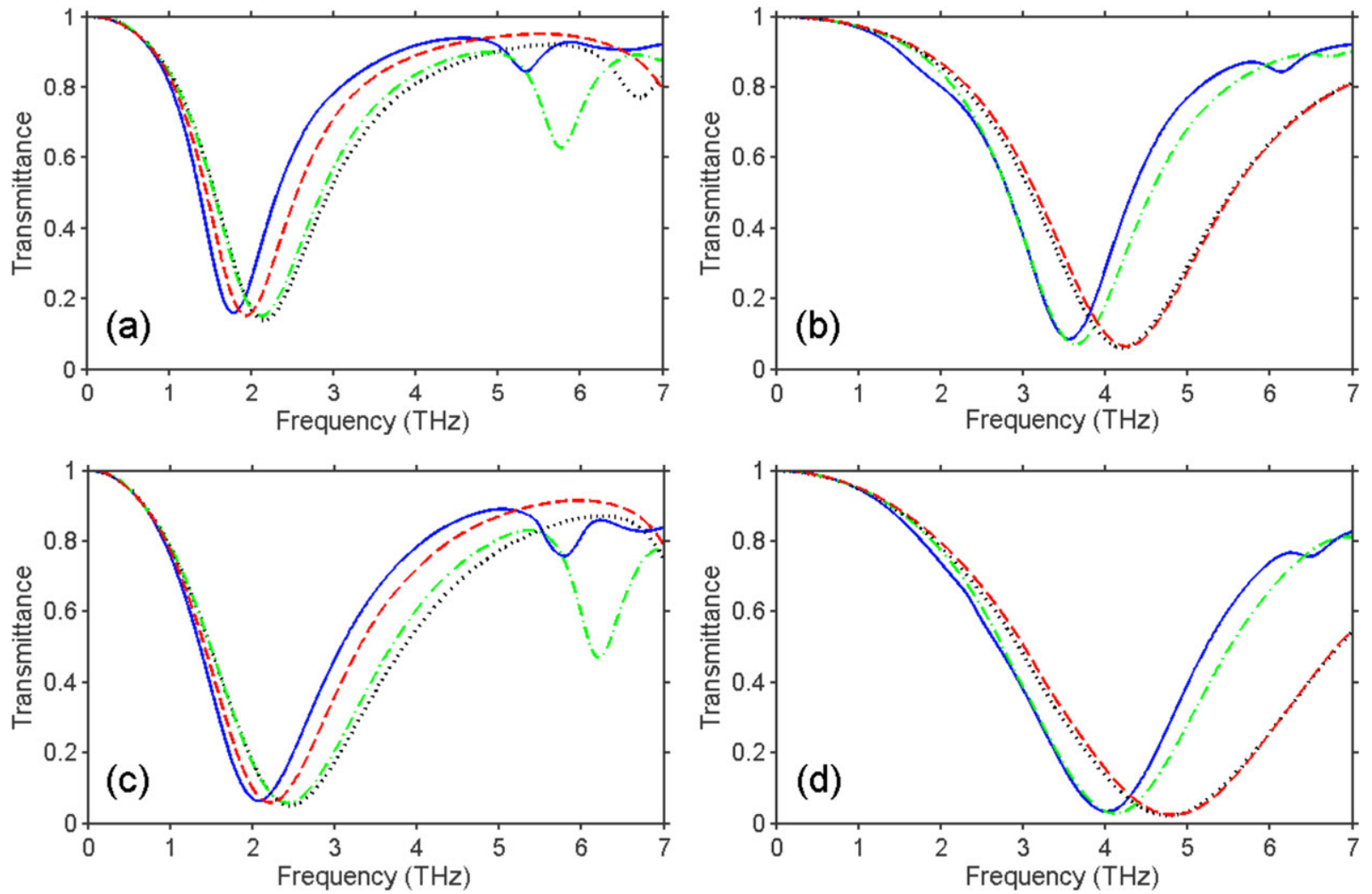

Figure 4. Computed spectra of a,c) $t_{x x}$ and $\left.b, d\right) t_{y y}$ for $\left.\mathrm{a}, \mathrm{b}\right) M=1$ and $\left.\mathrm{c}, \mathrm{d}\right) M=2$ with $b=1 \mu \mathrm{m}$, when $\varepsilon_{d}=1.0$ and $a=a_{0}$. Solid blue lines are for the Faraday configuration when $B_{0}=1 \mathrm{~T}$, dashed red lines for the Voigt-Y configuration when $B_{0}=1 \mathrm{~T}$, dash-dotted green lines for the Voigt-X configuration when $B_{0}=1 \mathrm{~T}$, and the dotted black line for the bias-free configuration $\left(B_{0}=0\right)$.
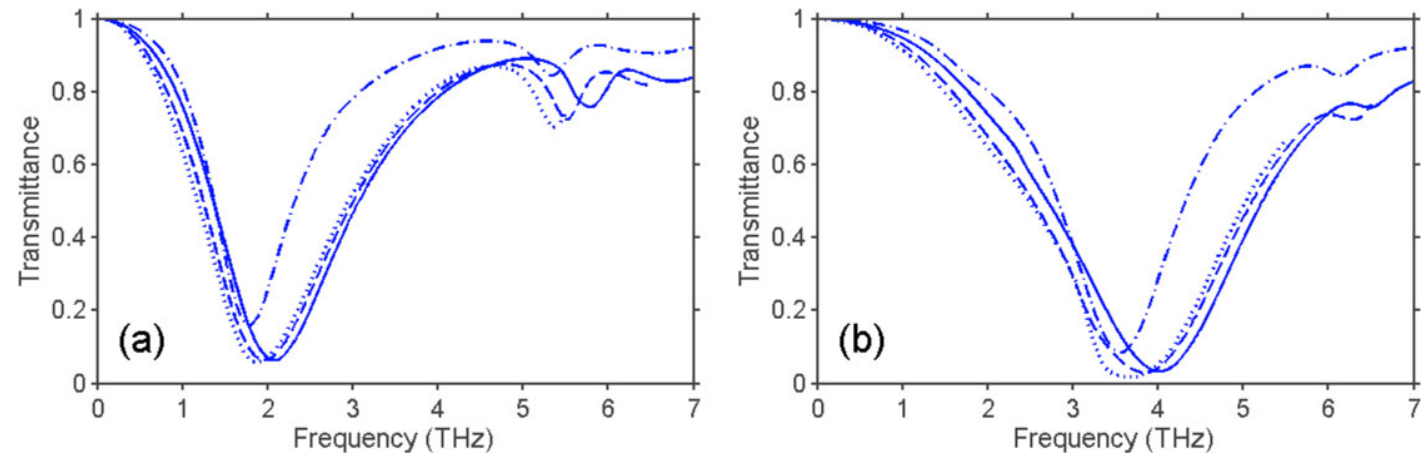

Figure 5. Computed spectra of a) $t_{x x}$ and b) $t_{y y}$ for the Faraday configuration when $B_{0}=1 \mathrm{~T}, \varepsilon_{d}=1$ and $a=a_{0}$. Dash-dotted lines are for $M=1$, solid lines for $M=2$ and $b=1 \mu \mathrm{m}$, dashed lines for $M=2$ and $b=2.5 \mu \mathrm{m}$, and dotted lines for $M=2$ and $b=5.5 \mu \mathrm{m}$.

phenomena for $M=1$ than are covered in Refs. [41-44]. We found that the cases with $b>5.5 \mu \mathrm{m}$ are not very useful for cascaded metasurfaces and therefore confined ourselves to $b \leq$ $5.5 \mu \mathrm{m}$.

Whereas the choice of $b$ definitely governs $f_{c}$, the effect of $b$ is weaker on the range $\Delta f_{c}^{(m)}$ obtainable by switching the magnetostatic field, i.e., by changing from $B_{0}=0$ to $B_{0}=1 \mathrm{~T}$ or vice versa, in the most sensitive (in our case, the Faraday) configuration when $M=2$. Thus, the values of $\Delta f_{c}^{(m)}$ are $0.38 \mathrm{THz}$ and $0.75 \mathrm{THz}$ for $\mathbf{E}_{\text {inc }} \| \hat{\mathbf{x}}$ and $\mathbf{E}_{\text {inc }} \| \hat{\mathbf{y}}$, respectively, when $b=1.0 \mu \mathrm{m}$; $0.39 \mathrm{THz}$ and $0.73 \mathrm{THz}$ when $b=2.5 \mu \mathrm{m}$; and $0.37 \mathrm{THz}$ and 0.65
THz when $b=5.5 \mu \mathrm{m}$. Hence, the effects of metasurface geometry and biasing field can be considered separately for design purposes, which is the third design principle.

All discussion in this section thus far has assumed that $a=a_{0}$. Tables 1 and 2 indicate that an increase of $a$ starting from $a_{0}$ still leads to variations in $f_{c}$, so that the choice of $a$ can be used as a design parameter to realize a specific stopband. This effect is not surprising in view of Floquet theory ${ }^{[45]}$ and the scale invariance of the Maxwell equations, ${ }^{[46,47]}$ subject of course to the frequency dependences of the constitutive parameters involved. Within the parameters of our investigation, the variation of $f_{c}$ with $a$ 
Table 1. Center frequency $f_{c}(\mathrm{THz})$ of the first stopband $\left(\mathrm{E}_{\mathrm{inc}} \| \hat{\mathbf{x}}\right)$ for $M=$ 1 and $M=2$ and specified values of $a$ and $b$, when $\varepsilon_{d}=1.0$ and $a_{0}=$ $15.56 \mu \mathrm{m}$.

\begin{tabular}{|c|c|c|c|c|}
\hline & Faraday, $\mathrm{B}_{0}=1 \mathrm{~T}$ & Voigt- $Y, B_{0}=1 \mathrm{~T}$ & Voigt- $X, B_{0}=1 \mathrm{~T}$ & $\mathrm{~B}_{0}=0$ \\
\hline \multicolumn{5}{|c|}{$M=1, a=a_{0}$} \\
\hline & 1.78 & 1.94 & 2.11 & 2.15 \\
\hline \multicolumn{5}{|c|}{$\mathrm{M}=2, \mathrm{a}=\mathrm{a}_{0}$} \\
\hline $\mathrm{b}=0.75 \mu \mathrm{m}$ & 2.11 & 2.24 & 2.45 & 2.49 \\
\hline $\mathrm{b}=1.0 \mu \mathrm{m}$ & 2.08 & 2.22 & 2.42 & 2.46 \\
\hline $\mathrm{b}=2.5 \mu \mathrm{m}$ & 1.96 & 2.10 & 2.31 & 2.35 \\
\hline $\mathrm{b}=5.5 \mu \mathrm{m}$ & 1.88 & 2.02 & 2.24 & 2.25 \\
\hline \multicolumn{5}{|c|}{$\mathrm{M}=2, \mathrm{a}=1.1 \mathrm{a}_{0}$} \\
\hline $\mathrm{b}=1.0 \mu \mathrm{m}$ & 2.30 & 2.45 & 2.67 & 2.71 \\
\hline \multicolumn{5}{|c|}{$\mathrm{M}=2, \mathrm{a}=1.25 \mathrm{a}_{0}$} \\
\hline $\mathrm{b}=1.0 \mu \mathrm{m}$ & 2.43 & 2.58 & 2.79 & 2.85 \\
\hline \multicolumn{5}{|c|}{$M=2, a=1.5 a_{0}$} \\
\hline $\mathrm{b}=1.0 \mu \mathrm{m}$ & 2.50 & 2.65 & 2.86 & 2.93 \\
\hline
\end{tabular}

Table 2. Center frequency $f_{c}(\mathrm{THz})$ of the second stopband (Einc $\left.\| \hat{\mathbf{y}}\right)$ for $M=1$ and $M=2$ and specified values of $a$ and $b$, when $\varepsilon_{d}=1.0$ and $a_{0}=15.56 \mu \mathrm{m}$.

\begin{tabular}{|c|c|c|c|c|}
\hline & Faraday, $\mathrm{B}_{0}=1 \mathrm{~T}$ & Voigt-Y, $B_{0}=1 \mathrm{~T}$ & Voigt- $X, B_{0}=1 \mathrm{~T}$ & $\mathrm{~B}_{0}=0$ \\
\hline \multicolumn{5}{|c|}{$M=1, a=a_{0}$} \\
\hline & 3.56 & 4.25 & 3.63 & 4.20 \\
\hline \multicolumn{5}{|c|}{$M=2, a=a_{0}$} \\
\hline $\mathrm{b}=0.75 \mu \mathrm{m}$ & 4.07 & 4.87 & 4.16 & 4.83 \\
\hline $\mathrm{b}=1.0 \mu \mathrm{m}$ & 4.01 & 4.81 & 4.11 & 4.76 \\
\hline $\mathrm{b}=2.5 \mu \mathrm{m}$ & 3.84 & 4.64 & 3.94 & 4.57 \\
\hline $\mathrm{b}=5.5 \mu \mathrm{m}$ & 3.67 & 4.42 & 3.80 & 4.32 \\
\hline \multicolumn{5}{|c|}{$\mathrm{M}=2, \mathrm{a}=1.1 \mathrm{a}_{0}$} \\
\hline $\mathrm{b}=1.0 \mu \mathrm{m}$ & 4.25 & 5.14 & 4.37 & 5.10 \\
\hline \multicolumn{5}{|c|}{$M=2, a=1.25 a_{0}$} \\
\hline $\mathrm{b}=1.0 \mu \mathrm{m}$ & 4.34 & 5.25 & 4.47 & 5.22 \\
\hline \multicolumn{5}{|c|}{$\mathrm{M}=2, \mathrm{a}=1.5 \mathrm{a}_{0}$} \\
\hline $\mathrm{b}=1.0 \mu \mathrm{m}$ & 4.36 & 5.26 & 4.50 & 5.24 \\
\hline
\end{tabular}

becomes smaller with increasing $a$ due to weaker coupling between the elements on the same metasurface; for $a$ in the neighborhood of $a_{0}$, the effect of coupling of this type is still significant.

An increase in $a$ and an increase in $b$ have opposite effects on $f_{c}$. Likewise, decreases in both $a$ and $b$ have opposite effects on $f_{c}$. In other words, inter-metasurface coupling and inter-resonator coupling on the same metasurface affect the spectral location of a stopband in opposite ways, which is the fourth design principle identified by us.

Generally, the performances of cascaded metasurfaces involve contributions from different effects whose identifications require detailed parametric investigations. ${ }^{[17]}$ We have correlated several selected features in the spatial profiles of the fields with the appearance of stopbands. These features mainly indicate field enhancement in certain portions of the unit cell, which include the regions between the two arms of the $\mathrm{H}$-shaped resonator, as well as in the close vicinity of and inside the arms. Although the presence of local enhancement does not always correspond to a strong reflection, it very often serves as a signature thereof. Indeed, strong confinement between the neighboring unit cells affects the efficiency of beam deflection by gradient metasurfaces. ${ }^{[17]}$

Moreover, the spatial profiles are dynamically changeable insofar as $\overline{\bar{\varepsilon}}$ is affected by the orientation and magnitude of the magnetostatic field. As an example, Figure 6 presents the spatial profiles for both linear polarization states (i.e., Einc $\| \hat{\mathbf{x}}$ and $\mathbf{E}_{\text {inc }} \| \hat{\mathbf{y}}$ ) of the incident plane wave at the frequency of the smallest reflectance in a stopband for $M=2$ and $\mathbf{E}_{\text {inc }} \| \hat{\mathbf{x}}$. When $\mathbf{E}_{\text {inc }} \| \hat{\mathbf{x}}$, the reflectance is high and the $\mathrm{H}$-shaped resonators in adjacent unit cells are strongly coupled to each other, the coupling being manifested by (i) strong electric fields in the gaps identified by A and (ii) strong magnetic fields in the regions identified by $\mathrm{C}$ in Figure 6 . When $\mathbf{E}_{\text {inc }} \| \hat{\mathbf{y}}$, the transmittance is moderate and the $\mathrm{H}$-shaped resonators in adjacent cells are strongly coupled to each other so that electric field is strong in the gaps identified by B in Figure 6 , but the magnetic field is not strongly affected by the structure. It is worth noting that the field components (including the ones parallel to $\hat{\mathbf{z}}$ ) that are absent in the incident plane wave, may nevertheless appear in the array. A deeper study that includes phase analysis is required to fully correlate specific features in the spatial profiles of the fields to the stopbands for specific polarization states of the incident plane wave and the magnetostatic-field configuration. We plan to report those correlations in due course of time.

\section{Resonance Scaling via Substrate/Spacer Permittivity}

The choice of a material with appropriate constitutive characteristics for the substrate/spacer is critical for fixing the stopband when a dynamic-control mechanism is not being deployed. ${ }^{[48-56]}$ In particular, scaling of resonances can be achieved with proper choice of $\varepsilon_{d}{ }^{[15]}$ In the general sense, this means that their spectral locations can be changed in a controllable manner, whereas other characteristics, e.g., field distribution and transmittances are preserved either partially or substantially. The main effects of a dielectric layer functioning either as a spacer between two metasurfaces or as a substrate for a single metasurface, as in this paper, are spectral shifts of the subwavelength resonances and related stopbands.

From classical electromagnetic theory we know that varying the relative permittivity $\varepsilon_{d}$ of a dielectric material filling a closed lossless cavity results in scaling of resonance frequencies by $\varepsilon_{d}^{-1 / 2[39]}$. Hence, replacing one dielectric material by another having larger/smaller $\varepsilon_{d}$ and occupying the same volume results in redshifts/blueshifts of resonance frequencies. These shifts can be quantified in terms of a scaling rule with respect to $\varepsilon_{d}$. The situation is different when we study open structures instead of the closed cavities. Indeed, in an open structure, at resonance the field may be strong outside the resonator.

This situation is quite typical of an array of subwavelength resonators on a dielectric substrate, as in Figure 1. This means that the resonance frequencies and the spatial profiles of the electromagnetic fields of a metasurface at any resonance frequency may be strongly affected by the substrate characteristics. This effect is 

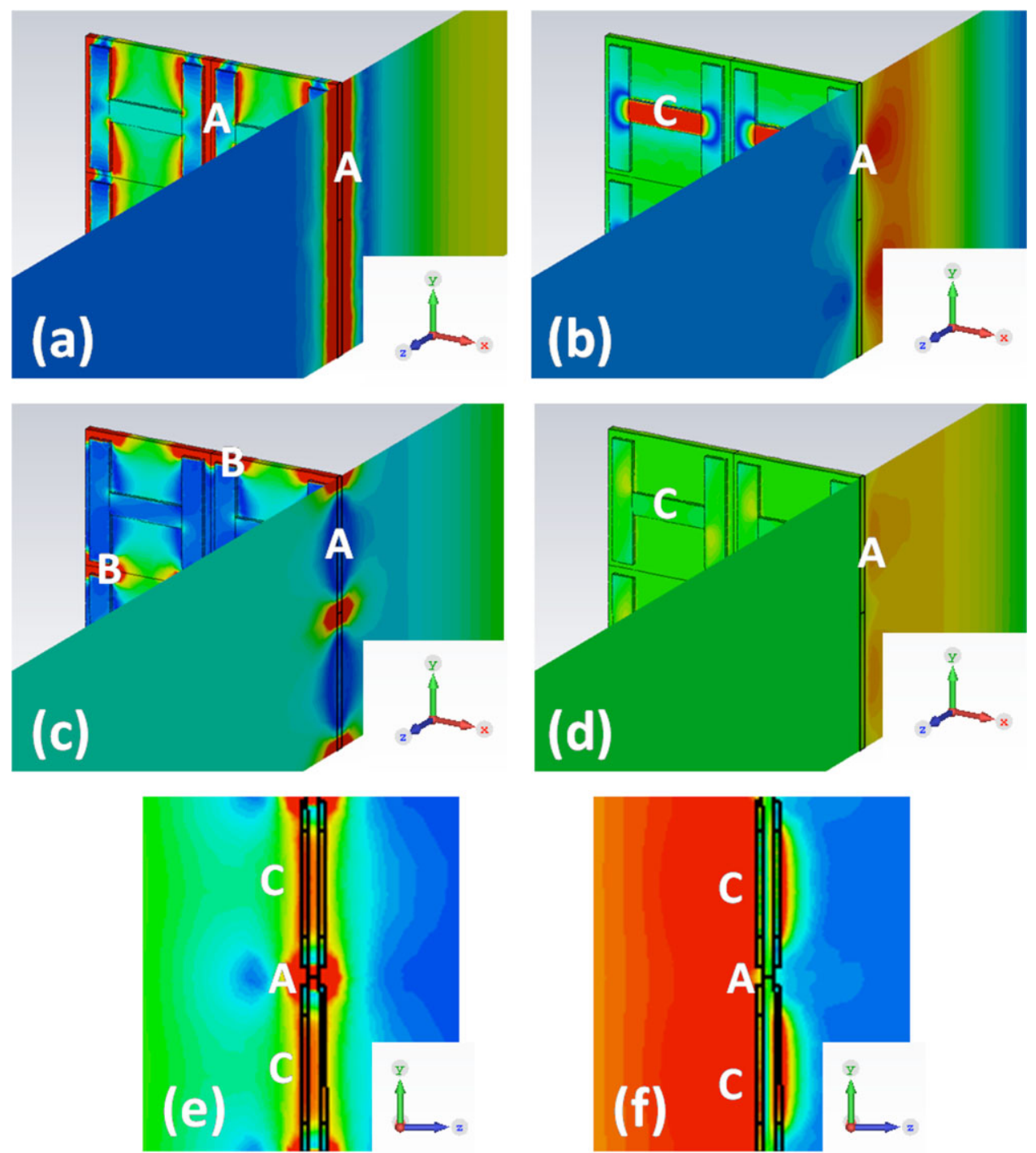

Figure 6. Spatial profiles of the electromagnetic fields at $2.08 \mathrm{THz}$ in a $2 \times 2$ array of unit cells, for $M=2, b=1 \mu \mathrm{m}, a=a_{0}$, and $\varepsilon_{d}=1.0$ when the magnetostatic field is in the Faraday configuration with $B_{0}=1 \mathrm{~T}$. a) $\left|E_{x}\right|$ and b) $\left|H_{y}\right|$ when $E_{\text {inc }} \| \hat{\mathbf{x}}$, the cutting plane being placed midway between the arms of two adjacent $\mathrm{H}$-shaped resonators; c) $\left|E_{y}\right|$ and d) $\left|H_{x}\right|$ when $E_{\text {inc }} \| \hat{\mathbf{y}}$, the cutting plane being placed midway between the arms of two adjacent $\mathrm{H}$-shaped resonators, $x=7.78 \mu \mathrm{m}$; e) $|\mathbf{E}|$ and $\mathrm{f})|\mathrm{H}|$, when $\mathrm{E}_{\text {inc }} \| \hat{\mathbf{x}}$, the cutting plane being at the middle of the horizontal arms of $\mathrm{H}$-shaped resonators, i.e., it coincides with the plane $y=0$.

asymmetric in the sense that the spatial profiles in the substrate away from the adjacent side of the metasurface differ from those in the neighboring free space away from the other side of the metasurface. The presence of a superstrate identical to the substrate will remove the asymmetry.

A partial analogy exists with a closed empty cavity into which we first introduce an electrically small dielectric body that perturbs the resonance frequency as well as the spatial profiles of the electromagnetic fields, ${ }^{[57,58]}$ and then increase the volume of the introduced body until it fills the whole cavity. If the dielectric body is placed initially at the location of a local maximum of the electric field, its effect on the cavity will be substantial ${ }^{[57,58]}$; the effect will continue to increase with the volume of the introduced body. A sparsely investigated issue is of the variations in resonance frequencies with gradual variation of that volume, i.e., when only a part of the cavity is kept unfilled. A similar but even more complicated situation occurs in the open-resonance structure that a metasurface is, as the spatial profiles of the fields in just a part of the original metasurface (i.e., the metasurface without a substrate) are affected by the dielectric substrate.

Scaling of resonance frequencies can indeed be achieved in open resonance structures with the capability to manipulate the polarization state by changing only the constitutive properties of the substrate/spacer, while all dimensions are fixed. As an example, the variation of $f_{c}$ of the type $\varepsilon_{d}^{-\alpha}$ has been demonstrated for $0.36<\alpha<0.45,^{[15]}$ quite close to $\alpha=0.5$ required by the classical rule. ${ }^{[39]}$ What is even more interesting is that the secondary electromagnetic characteristics, such as the polarization-conversion efficiency can be preserved while varying $\varepsilon_{d}$ in a wide range. ${ }^{[15]}$ However, it remains unclear whether this situation is typical also for other types of the structures comprising subwavelength resonators. For the sake of completeness, we must mention the 

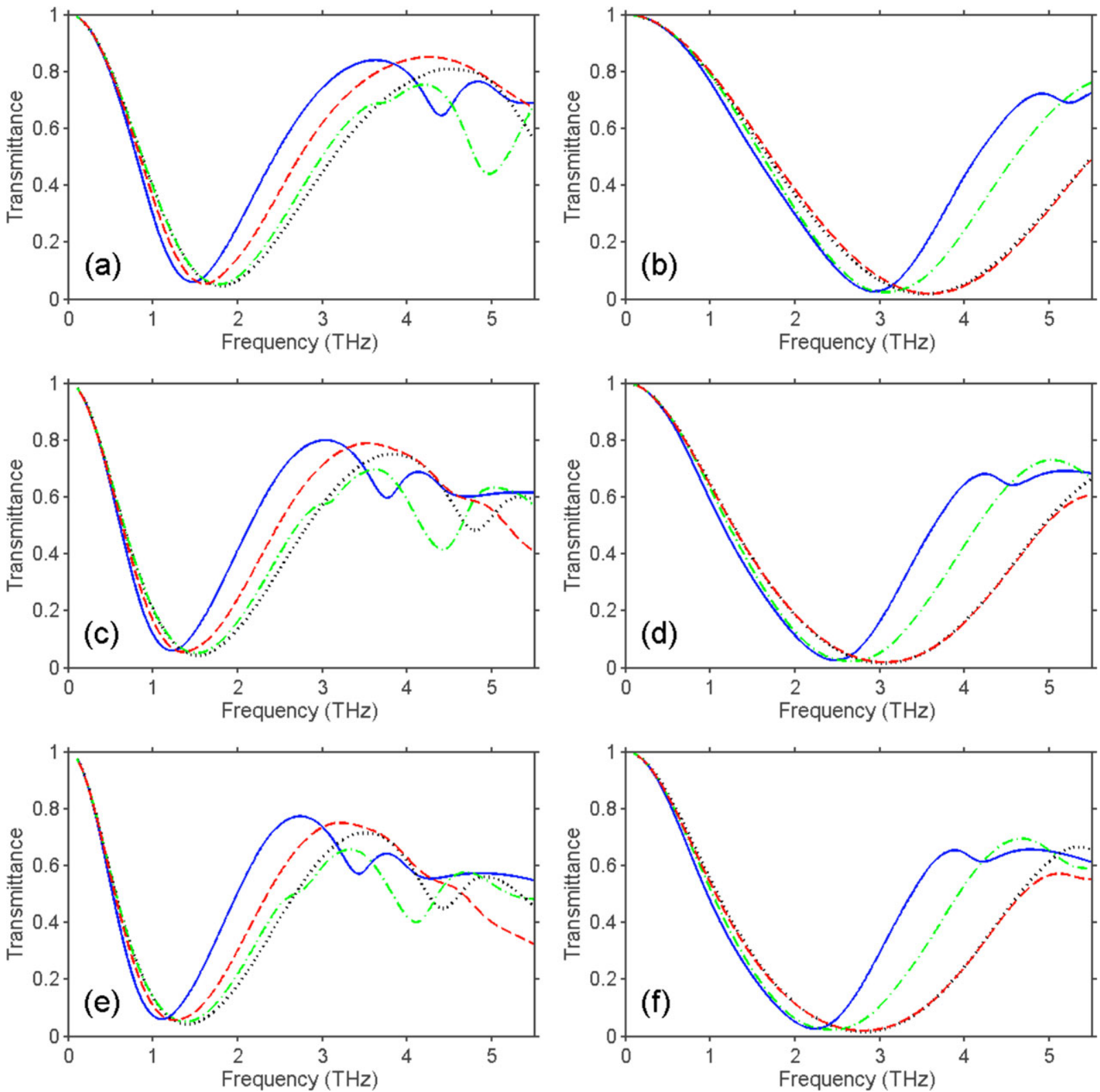

Figure 7. Computed spectra of a,c,e) $t_{x x}$ and b,d,f) $t_{\gamma \psi}$ for $M=2, b=1 \mu \mathrm{m}$, and $a=a_{0}$, when a,b) $\varepsilon_{d}=5.8$, c,d) $\varepsilon_{d}=9.6$, and e,f) $\varepsilon_{d}=12.2$. Solid blue lines are for the Faraday configuration when $B_{0}=1 \mathrm{~T}$, dashed red lines for the Voigt-Y configuration when $B_{0}=1 \mathrm{~T}$, dash-dotted green lines for the Voigt-X configuration when $B_{0}=1 \mathrm{~T}$, and the dotted black line for the bias-free configuration $\left(B_{0}=0\right)$.

commonly used way of scaling is based on proportional changes of all geometrical and constitutive parameters, ${ }^{[46,47]}$ which may not always be straightforward to implement because the constitutive parameters of any material are frequency dependent.

Thus, resonance scaling means that (i) resonances and related stopbands remain, (ii) their shifts can be parameterized, and (iii) transmission and polarization characteristics are mostly preserved, when $\varepsilon_{d}$ is varied but all dimensions are kept fixed. We must also bear in mind that changing $\varepsilon_{d}$ while keeping $b$ fixed may affect inter-metasurface coupling, which may affect the stopband spectrum if $M>1$.

In order to explore resonance scaling, the calculations for Figure $4(\mathrm{c}, \mathrm{d})$ were repeated for $a=a_{0}, b=1 \mu \mathrm{m}$, and $M=2$, but for three different values of $\varepsilon_{d}: 5.8$ (typical of chalcogenide glasses), 9.6 (typical of aluminum-oxide-based composites), and 12.2 (silicon). The results provided in Figure 7 indicate that the stopbands for $\varepsilon_{d}>1$ are redshifted and compressed analogs of the stopbands presented in Figure $4(\mathrm{c}, \mathrm{d})$ for $\varepsilon_{d}=1$. Neither the depths of the stopbands nor the dependences on the magnetostatic-field configuration are significantly affected by increasing $\varepsilon_{d}$ in the range $[1,12.2]$.

Next, we increased the spacer thickness to $b=2.5 \mu \mathrm{m}$ while keeping $a=a_{0}$ and $M=2$ fixed, and we recalculated the transmittance spectra with the same three values of $\varepsilon_{d}$ as in Figure 7. The results are presented for this case in Figure 8. All the features observed for $b=1 \mu \mathrm{m}$ in Figure 7 , including those related to 

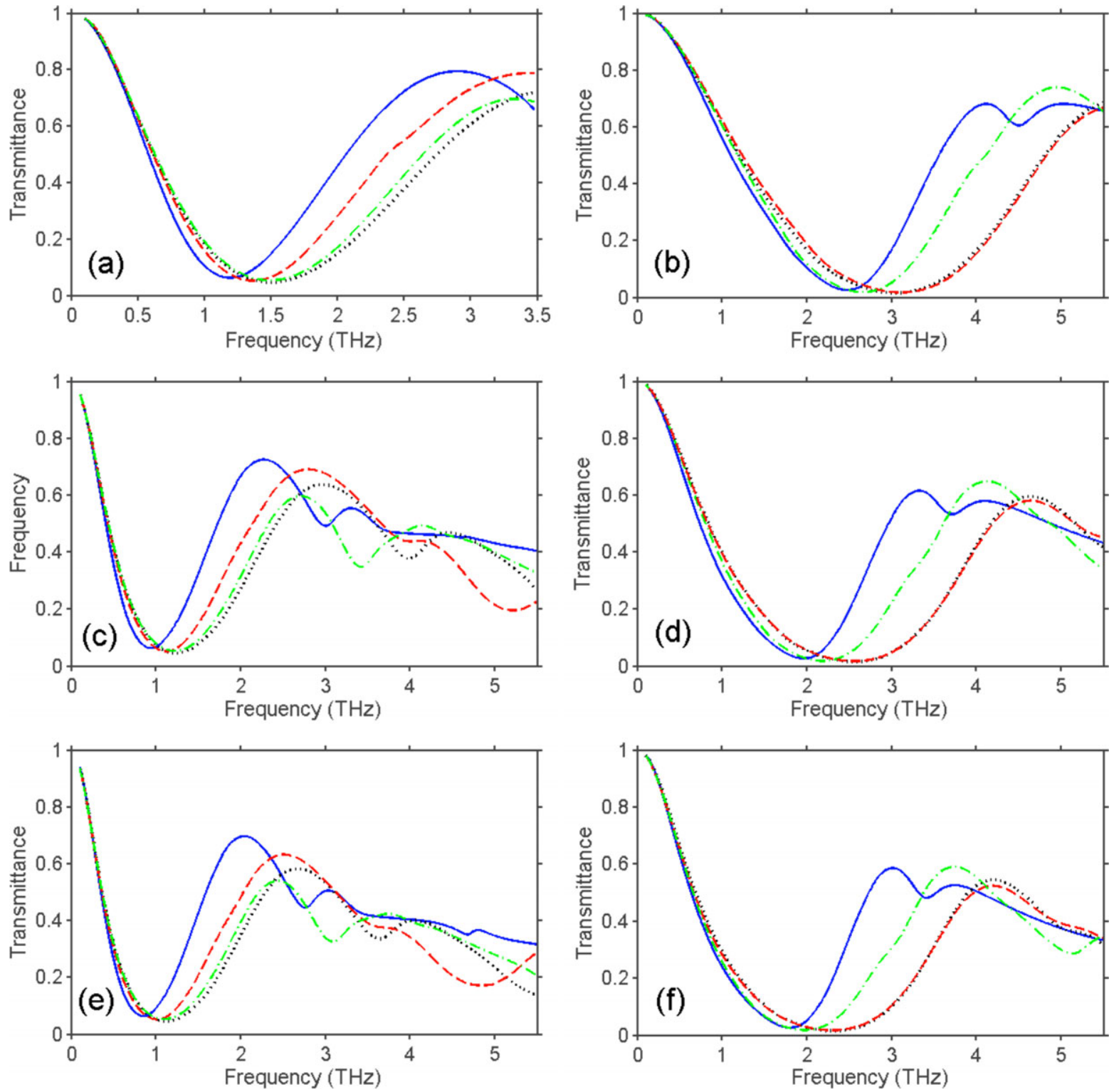

Figure 8. Same as Fig. 7 but for $b=2.5 \mu \mathrm{m}$.

switching and tuning by means of magnetostatic-field variations, are also found for $b=2.5 \mu \mathrm{m}$ in Figure 8, indicating that the spacer thickness does not qualitatively affect the intermetasurface coupling mechanism. Rather, its main effect is related to shifts of $f_{c}$, at least for relatively small values of $b$ for which Fabry-Perot resonances are not expected to appear in the spacer. In principle, Fabry-Perot resonances can be used but are expected to be impractical because of large thickness of the resulting structure.

Values of $f_{c}$ of the first and second stopbands for $M=1, b \in$ $\{1,2.5\} \mu \mathrm{m}, a=15.56 \mu \mathrm{m}$, and $\varepsilon_{d} \in\{1,5.8,9.6,12.2\}$ were examined in order to quantify resonance scaling, the data being provided in Supporting Information Tables S1 and S2. Analogous data for $M=2, b \in\{1,2.5,5.5\} \mu \mathrm{m}, a=15.56 \mu \mathrm{m}$, and $\varepsilon_{d} \in\{1,5.8,9.6,12.2\}$ in Supporting Information Tables S3 and S4 were also examined. Approximate fitting of these data to $f_{c} \propto$ $\varepsilon_{d}^{-\alpha}$ yielded $0.22<\alpha<0.4$. Thus, the deviation $\delta=|\alpha-0.5|$ from the classical scaling rule can be quite significant. In addition to its dependence on $M$, the value of $\alpha$ depends on $b$, thereby indicating the influence on the thickness of the substrate/spacer. Besides, $\alpha$ depends on the polarization state of the incident plane wave as well as on the magnetostatic-field configuration. Larger values of $\alpha$ are observed for the Faraday configuration and for Einc $\| \hat{\mathbf{x}}$.

For fixed values of $M, a$, and $b$, the relationship $f_{c} \propto \varepsilon_{d}^{-\alpha}$ with the fitted value of $\alpha$ underestimates $f_{c}$ except near the boundaries of the range $1<\varepsilon_{d}<12.2$, where the relationship predicts $f_{c}$ very well. So, strictly speaking, a different relationship should be sought, but the $\varepsilon_{d}^{-\alpha}$ trend is very appropriate for qualitative comparison with the classical trend $\varepsilon_{d}^{-1 / 2}$. For the Faraday configuration, Figure 9 presents the results calculated with $\varepsilon_{d}=$ 5.8 and $M=2$ for several different values of $b$. Comparing 

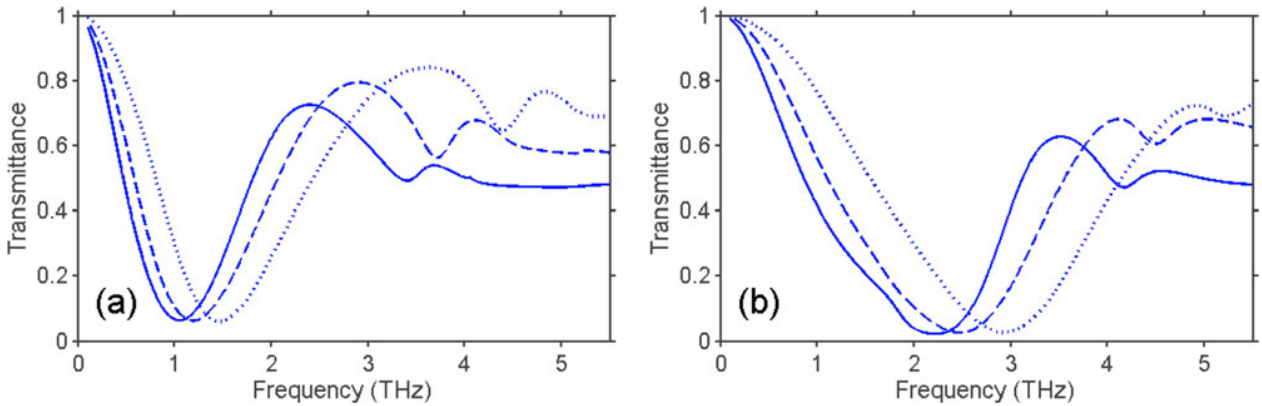

Figure 9. Computed spectra of a) $t_{x x}$ and b) $t_{y y}$ when $M=2$ and $\varepsilon_{d}=5.8$, for the Faraday configuration with $B_{0}=1 \mathrm{~T}$. Dotted lines are for $b=1 \mu \mathrm{m}$ dashed lines are for $b=2.5 \mu \mathrm{m}$, and solid lines are for $b=5.5 \mu \mathrm{m}$.

Table 3. Range $\Delta f_{c}^{\left(\varepsilon_{d}\right)}(\mathrm{THz})$ of $f_{c}$ as $\varepsilon_{d}$ varies from 1 to 12.2 , when $a=$ $15.56 \mu \mathrm{m}$.

\begin{tabular}{|c|c|c|c|c|c|c|}
\hline & \multicolumn{3}{|c|}{ First Stopband $\left(\mathrm{E}_{\text {inc }} \| \hat{\mathbf{x}}\right), t_{x x}$} & \multicolumn{3}{|c|}{ Second Stopband ( $\left.E_{\text {inc }} \| \hat{\mathbf{y}}\right), t_{\gamma y}$} \\
\hline & \multicolumn{6}{|c|}{$b=1 \mu \mathrm{m} \quad b=2.5 \mu \mathrm{m} \quad b=5.5 \mu \mathrm{m}$} \\
\hline \multicolumn{7}{|c|}{$M=1$} \\
\hline $\begin{array}{l}\text { Faraday, } \\
\qquad B_{0}=1 \mathrm{~T}\end{array}$ & 0.96 & 1.09 & - & 1.80 & 2.01 & - \\
\hline $\begin{array}{l}\text { Voigt-Y, } \\
\qquad B_{0}=1 \mathrm{~T}\end{array}$ & 0.91 & 1.08 & - & 2.0 & 2.31 & - \\
\hline $\begin{array}{l}\text { Voigt-X, } \\
\qquad B_{0}=1 \mathrm{~T}\end{array}$ & 1.0 & 1.19 & - & 1.68 & 1.94 & - \\
\hline$B_{0}=0$ & \multicolumn{5}{|c|}{$M=2$} & - \\
\hline $\begin{array}{l}\text { Faraday, } \\
\qquad B_{0}=1 \mathrm{~T}\end{array}$ & 0.98 & 1.11 & 1.16 & 1.79 & 2.04 & 2.09 \\
\hline $\begin{array}{l}\text { Voigt-Y, } \\
\qquad B_{0}=1 \mathrm{~T}\end{array}$ & 0.97 & 1.09 & 1.13 & 2.02 & 2.35 & 2.36 \\
\hline $\begin{array}{l}\text { Voigt-X, } \\
\qquad B_{0}=1 \mathrm{~T}\end{array}$ & 1.04 & 1.22 & 1.28 & 1.88 & 1.97 & 2.05 \\
\hline$B_{0}=0$ & 1.06 & 1.23 & 1.27 & 1.97 & 2.26 & 2.28 \\
\hline
\end{tabular}

Figures 5 and 9 , we can see that the effect of $b$ becomes stronger when $\varepsilon_{d}$ is increased.

The data in Tables 3 and $\mathbf{4}$ illustrate that dynamic tunability quantified by $\Delta f_{c}^{(m)}$ can be achieved by switching the magnetostatic field and varying the orientation of $\mathbf{B}_{0}$, whereas the value of $\varepsilon_{d}$ in the range $\Delta f_{c}^{\left(\varepsilon_{d}\right)}$ can be selected during the design phase with the parameters $M, a$, and $b$ fixed; furthermore, the ranges $\Delta f_{c}^{(m)}$ and $\Delta f_{c}^{\left(\varepsilon_{d}\right)}$ are connected with resonance scaling. The corresponding values of $f_{c}$ are given in Supporting Information Tables S1-S4. A comparison of the values of $\Delta f_{c}^{\left(\varepsilon_{d}\right)}$ in Table 3, which were calculated for $\varepsilon_{d}$ varying from 1 to 12.2 , indicates that a higher sensitivity to changes in $\varepsilon_{d}$ exists for $M=2$ than for $M=1$, and that this sensitivity is enhanced when $b$ is increased. This again confirms that a thicker substrate/spacer will affect the resonances more, and that this effect can be enhanced by intermetasurface coupling. There is a strong difference in the range $\Delta f_{c}^{\left(\varepsilon_{d}\right)}$ achievable for $t_{x x}$ and $t_{y \gamma}$, the difference being the strongest for the Voigt-Y configuration among the four magnetostatic-field configurations chosen for this work. In Table 4, one can see that
Table 4. Range $\Delta f_{c}^{(m)}(\mathrm{THz})$ of $f_{c}$ achievable by switching from the biasfree to the Faraday configuration, when $a=15.56 \mu \mathrm{m}$.

\begin{tabular}{|c|c|c|c|c|c|c|}
\hline & \multicolumn{3}{|c|}{ First Stopband $\left(E_{\text {inc }} \| \hat{\mathbf{x}}\right), t_{x x}$} & \multicolumn{3}{|c|}{ Second Stopband $\left(\boldsymbol{E}_{\text {inc }} \| \hat{\mathbf{y}}\right), t_{y y}$} \\
\hline & $b=1 \mu \mathrm{m}$ & $=2.5 \mu \mathrm{m}$ & $b=5.5 \mu \mathrm{m}$ & $b=1 \mu \mathrm{m}$ & $=2.5 \mu \mathrm{m}$ & $b=5.5 \mu \mathrm{m}$ \\
\hline \multicolumn{7}{|c|}{$M=1$} \\
\hline$\varepsilon_{\mathrm{d}}=1.0$ & 0.37 & 0.37 & 0.37 & 0.64 & 0.64 & 0.64 \\
\hline$\varepsilon_{\mathrm{d}}=2.1$ & 0.35 & 0.34 & - & 0.60 & 0.58 & - \\
\hline$\varepsilon_{\mathrm{d}}=5.8$ & 0.32 & 0.31 & - & 0.51 & 0.51 & - \\
\hline$\varepsilon_{\mathrm{d}}=9.6$ & 0.31 & 0.28 & - & 0.51 & 0.45 & - \\
\hline$\varepsilon_{\mathrm{d}}=12.2$ & 0.32 & 0.29 & - & 0.53 & 0.42 & - \\
\hline \multicolumn{7}{|c|}{$M=2$} \\
\hline$\varepsilon_{\mathrm{d}}=1.0$ & 0.38 & 0.39 & 0.37 & 0.75 & 0.73 & 0.65 \\
\hline$\varepsilon_{\mathrm{d}}=2.1$ & 0.37 & 0.35 & 0.35 & 0.69 & 0.66 & 0.63 \\
\hline$\varepsilon_{\mathrm{d}}=5.8$ & 0.34 & 0.31 & 0.30 & 0.62 & 0.58 & 0.53 \\
\hline$\varepsilon_{\mathrm{d}}=9.6$ & 0.31 & 0.30 & 0.28 & 0.58 & 0.58 & 0.50 \\
\hline$\varepsilon_{\mathrm{d}}=12.2$ & 0.30 & 0.27 & 0.26 & 0.57 & 0.51 & 0.46 \\
\hline
\end{tabular}

the range $\Delta f_{c}^{(m)}$ achieved dynamically between the bias-free and Faraday configurations for fixed $\varepsilon_{d}$ is usually narrower than the range $\Delta f_{c}^{\left(\varepsilon_{d}\right)}$ achieved at the design phase by means of $\varepsilon_{d}$ variation for a fixed configuration of magnetostatic field. In wide ranges of variation of $\varepsilon_{d}, b, M$, their effect on $\Delta f_{c}^{(m)}$ is rather weak. For the second stopband $\left(\mathrm{E}_{\text {inc }} \| \hat{\mathbf{y}}\right)$, the sensitivity of $\Delta f_{c}^{(m)}$ to the variations in $\varepsilon_{d}$ can be significant.

The obtained results show that resonance scaling takes place in the manner defined earlier in this section. Accordingly, choices of subwavelength resonators, arrays, substrate/spacer, and the magnetostatic-field configuration can be made separately, which should be considered as very desirable by metasurface designers. Indeed, the separability of these choices is the fifth principle for metasurface design.

Alongside the redshifts of the center frequencies of stopbands with increasing $\varepsilon_{d}$, resonance scaling compresses the stopbands in the $f$ domain so that their edges become steeper. However, it can also lead to unwanted reflections and reduced transmittances in the high-frequency neighborhood of a stopband. Moderate values of $\varepsilon_{d}$ can be optimal as the trade-off between compactness and transmission efficiency above the stopband, while substrate/spacer thickness should be chosen according to specific design requirements. If simultaneous manipulation of 
(a)
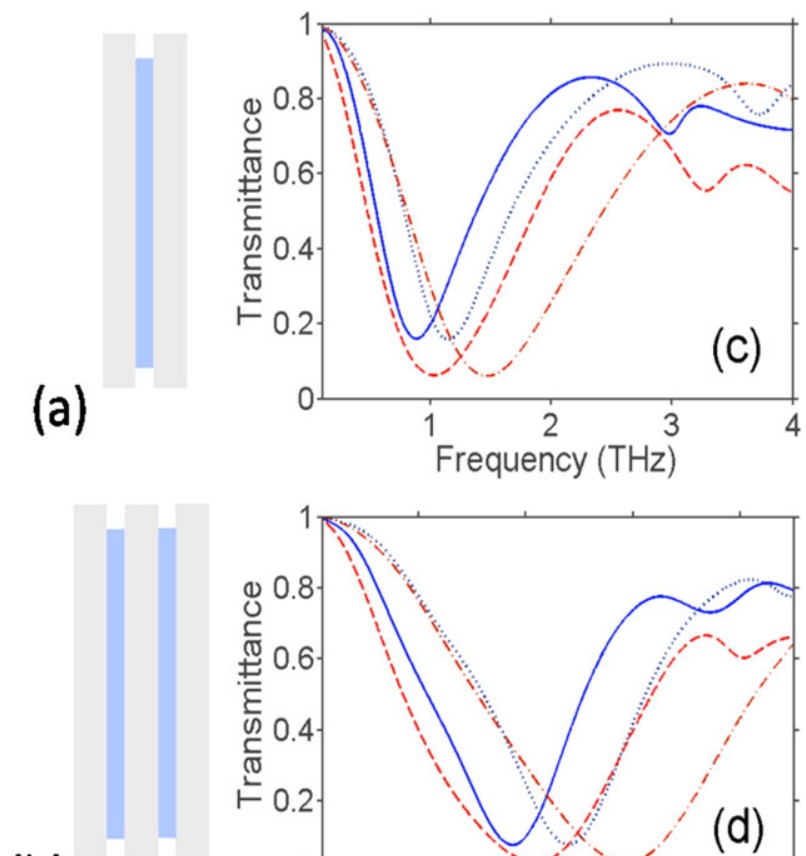

(b)

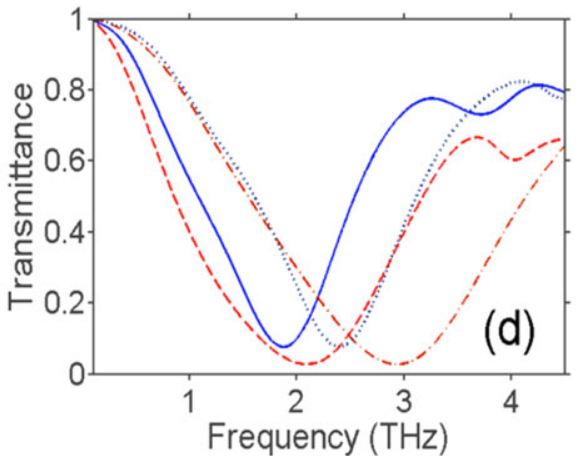

Figure 10. Schematics of the structures for a) $M=1$ and b) $M=2$ with a superstrate each. Computed spectrums of c) $t_{x x}$ and d) $t_{y y}$ for the Faraday configuration with $B_{0}=1 \mathrm{~T}$, when $a=15.56 \mu \mathrm{m}, b=1 \mu \mathrm{m}$, and $\varepsilon_{d}=5.8$. Solid blue lines are for $M=1$ and dashed red lines are for $M=2$, when the superstrate is present. Dotted blue lines are for $M=1$ and dash-dotted red lines are for $M=2$, when the superstrate is absent.

two polarization states of the incident plane wave by one device is unnecessary, the design parameters can be less constrained. Substrates/spacers with $\varepsilon_{d} \geq 6$ and $b \geq 5.5 \mu \mathrm{m}$ are not recommended, based on our calculations.

In addition to the possibility of separation of geometrical, constitutive, and biasing-field-related stages of the design process, we must also consider the transmittances in the lower-frequency and the higher-frequency neighborhoods of a stopband. Indeed, as seen in Figures 7 and 8, transmission in the lower-frequency neighborhood is quite robust with respect to the magnitude and orientation of $\mathbf{B}_{0}$, but transmission in the higher-frequency neighborhood is strongly sensitive to $\mathbf{B}_{0}$. We also note the weak difference in transmission between the Voigt- $X$ and bias-free configurations for $\mathbf{E}_{\text {inc }} \| \hat{\mathbf{x}}$, and between the Voigt-Y and bias-free configurations for $\mathbf{E}_{\text {inc }} \| \hat{\mathbf{y}}$.

When $\mathbf{E}_{\text {inc }} \| \hat{\mathbf{y}}$, Figures 7 and 8 indicate that one can realize an intermediate ON state between an ON state and an OFF state. One can first switch from the bias-free (OFF state) to either one of the two Voigt configurations (intermediate ON state), and then from the latter to the Faraday configuration (final ON state). This two-step switching scheme comes with freedom to choose one of two transmission regimes that differ in efficiency. Obtaining an intermediate $\mathrm{ON}$ state that is nearly in the middle between $t_{Y Y}^{(F)}$ and $t_{Y Y}{ }^{(0)} \sim 0$, i.e., either $t_{Y Y}^{(V X)} \approx t_{Y Y}^{(F)} / 2$ or $t_{Y Y}^{(V Y)} \approx t_{Y Y}^{(F)} / 2$, would be desirable. When $\mathbf{E}_{\text {inc }} \| \hat{\mathbf{x}}$, the contrast between $t_{x x}^{(F)}$ and $t_{x x}{ }^{(0)}$ can be insufficient to realize such a two-step switching scheme.
Finally, Figure 10 presents the results for the structures similar to those in Figures 1 and 7, but with a superstrate that has the same relative permittivity and thickness as the substrate/spacers. The structure's total thickness is $D=2 b+t$ when $M=1$ and $D=M(b+t)+b$ when $M>1$. The addition of the superstrate leads to a significant redshift of $f_{c}$. In particular, for $M=1$ and $M=2$, respectively, the center frequency of the prominent first stopband reduces by $0.52 \mathrm{THz}$ and $0.84 \mathrm{THz}$ when $\mathbf{E}_{\text {inc }} \| \hat{\mathbf{y}}$. Hence, the superstrate allows flexibility in engineering $f_{c}$ without affecting the stopband's depth.

\section{Cascading of $\mathbf{M}>2$ Metasurfaces}

Now, let us consider the transmittance spectra obtainable by cascading $M>2$ metasurfaces. Figure 11 presents the spectra of $t_{x x}$ and $t_{y y}$ analogous to those in Figure 7 but for $M=4$ (as shown in Figure 1(d)). In addition, we fixed $b=0.5 \mu \mathrm{m}$ instead of $b=1 \mu \mathrm{m}$ in order to keep the total thickness of the same order as for Figure 7. For comparison, the results for $M=4$ and $b=1 \mu \mathrm{m}$ are presented in Supporting Information Figure S7. As expected, ${ }^{[10]}$ the stopbands become deeper than for $M \in\{1,2\}$. There is more sensitivity in the Voigt-X and Voigt- $Y$ configurations to ON-OFF switching and the orientation of $\mathbf{B}_{0}$ in the high-frequency neighborhoods of the stopbands, as can be seen by comparing Figure 11 with Figures 7 and 8 . The center frequency $f_{c}$ of each stopband for $M=4$ and $b=0.5 \mu \mathrm{m}$ is blueshifted compared to the same stopband for $M=2$ and $b=1 \mu \mathrm{m}$, regardless of the magnetostatic-field configuration, for both $\varepsilon_{d}=5.8$ and $\varepsilon_{d}=12.2$. On the contrary, $f_{c}$ is redshifted for most of stopbands for $M=4$ and $b=1 \mu \mathrm{m}$. Generally, the effects on $f_{c}$ of varying $M$ and $\varepsilon_{d}$ run in either the same or opposite directions and can be used to either enhance or counteract each other. In addition, one can obtain a stopband with a desired center frequency and depth by playing with the values of $b$ and $M$. However, if one needs high transmittance in the high-frequency neighborhood of a stopband, structures with smaller $b$ and $M$ may be preferable due to weaker reflections from the dielectric layers. For further evidence of the effects of cascading, we have presented the results for $M \in\{1,2,4,8\}$ in Supporting Information Tables S5 and S6 for all four magnetostatic-field configurations, when $b \in\{1,2.5\} \mu \mathrm{m}, a=15.56 \mu \mathrm{m}$, and $\varepsilon_{d} \in\{5.8,12.2\}$. For comparison, the results for $b=0.5 \mu \mathrm{m}$ are also presented therein.

Figure 12 provides the transmittance spectra for the Faraday and bias-free configurations for $M \in\{1,2,4,8\}, b=1 \mu \mathrm{m}$, and $\varepsilon_{d}=5.8$, these two configurations being usually sufficient to estimate transmittance characteristics for the Voigt-X and Voigt- $Y$ configurations. The basic features for $B_{0}=1 \mathrm{~T}$ in the Faraday configuration are inherited from the bias-free configuration. A noticeable trend is the narrowing of the stopbands for all four values of $M$ when $B_{0} \neq 0$, regardless of $\varepsilon_{d}$ and the polarization state of the incident plane wave.

\section{Concluding Remarks}

To summarize, we considered the basic principles of cascading magnetostatically tunable metasurfaces which comprise 

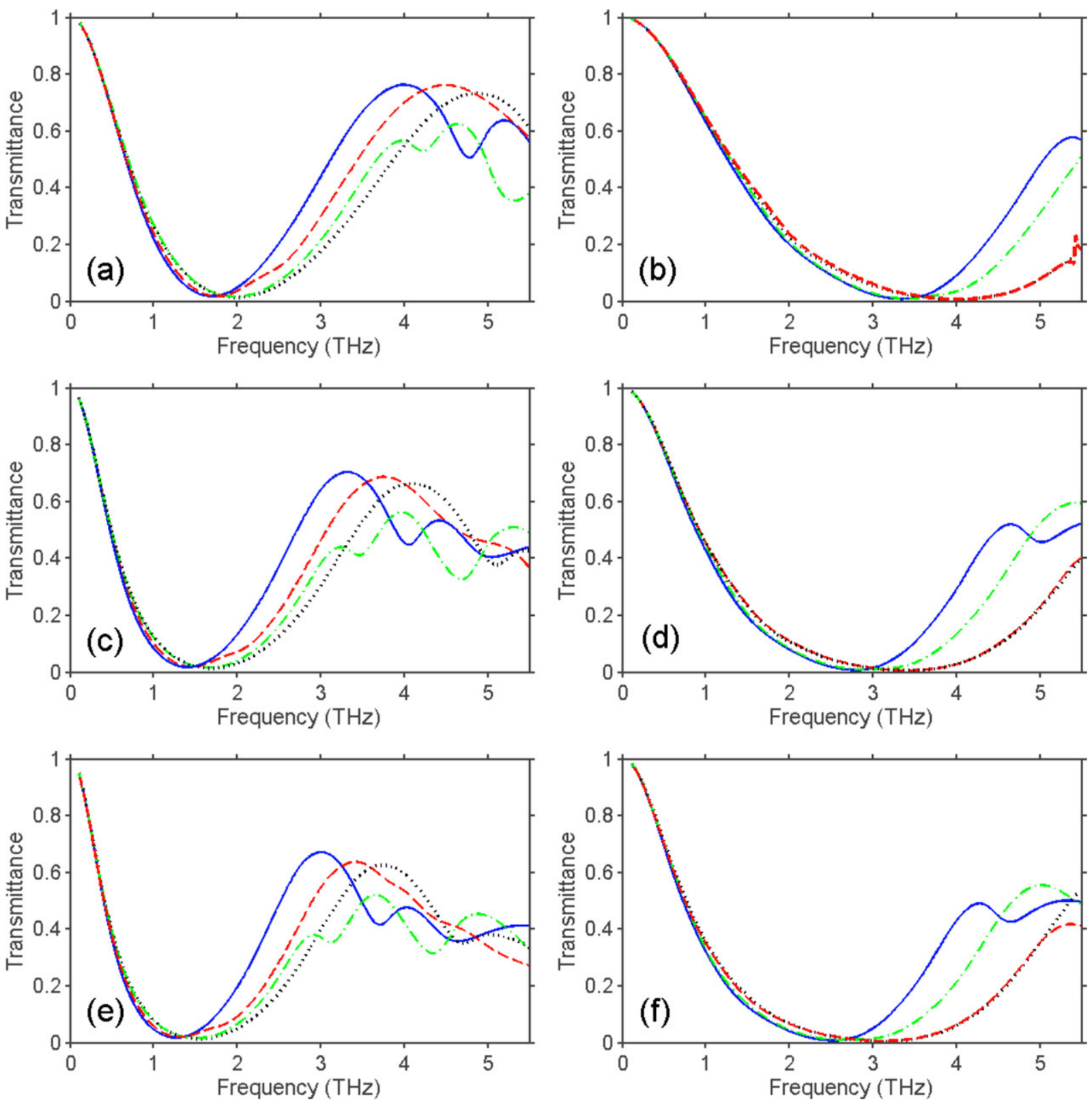

Figure 11. Computed spectra of a,c,e) $t_{x x}$ and b,d,f) $t_{\gamma y}$ for $M=4, b=0.5 \mu \mathrm{m}$, and $a=a_{0}$, when a,b) $\varepsilon_{d}=5.8$, c,d) $\varepsilon_{d}=9.6$, and e,f) $\varepsilon_{d}=12.2$. Solid blue lines are for the Faraday configuration when $B_{0}=1 \mathrm{~T}$, dashed red lines for the Voigt-Y configuration when $B_{0}=1 \mathrm{~T}$, dash-dotted green lines for the Voigt-X configuration when $B_{0}=1 \mathrm{~T}$, and the dotted black line for the bias-free configuration $\left(B_{0}=0\right)$.

$\mathrm{H}$-shaped InAs resonators. The coupling of two metasurfaces with an air spacer can be strongly modified by varying the distance $b$ between them, regardless of the studied orientations and magnitudes of the magnetostatic field $\mathbf{B}_{0}$. The inter-metasurface coupling as well as the coupling between the resonators on the same metasurface can be used for efficient tuning of the resonances and the related stopbands. At the same time, the width of the tunability range realizable due to variations of the magnetostatic-field orientation depends on $b$ relatively weakly.

The relative permittivity $\varepsilon_{d}$ of the dielectric substrate/spacer may strongly affect the resonances and the related stopbands.
The range of tunability of the center frequency $f_{c}$ which can be realized by variations in the magnetostatic-field orientation depends on $\varepsilon_{d}$. Typically, this range is wider for smaller values of $\varepsilon_{d}$, and the stopbands become narrower and redshift as $\varepsilon_{d}$ is increased. Moreover, the tunability range realizable by varying $\varepsilon_{d}$ depends on $b$, provided the magnetostatic-field orientation is fixed.

The spectral shifts of the center frequencies of the stopbands on varying $\varepsilon_{d}$ for fixed $\mathbf{B}_{0}$ are usually stronger than those due to variations of $\mathbf{B}_{0}$ for fixed $\varepsilon_{d}$. As expected, a stronger effect of $\varepsilon_{d}$ on the center frequencies of the stopbands is observed for thicker substrate/spacers. This effect occurs since a larger portion of the 

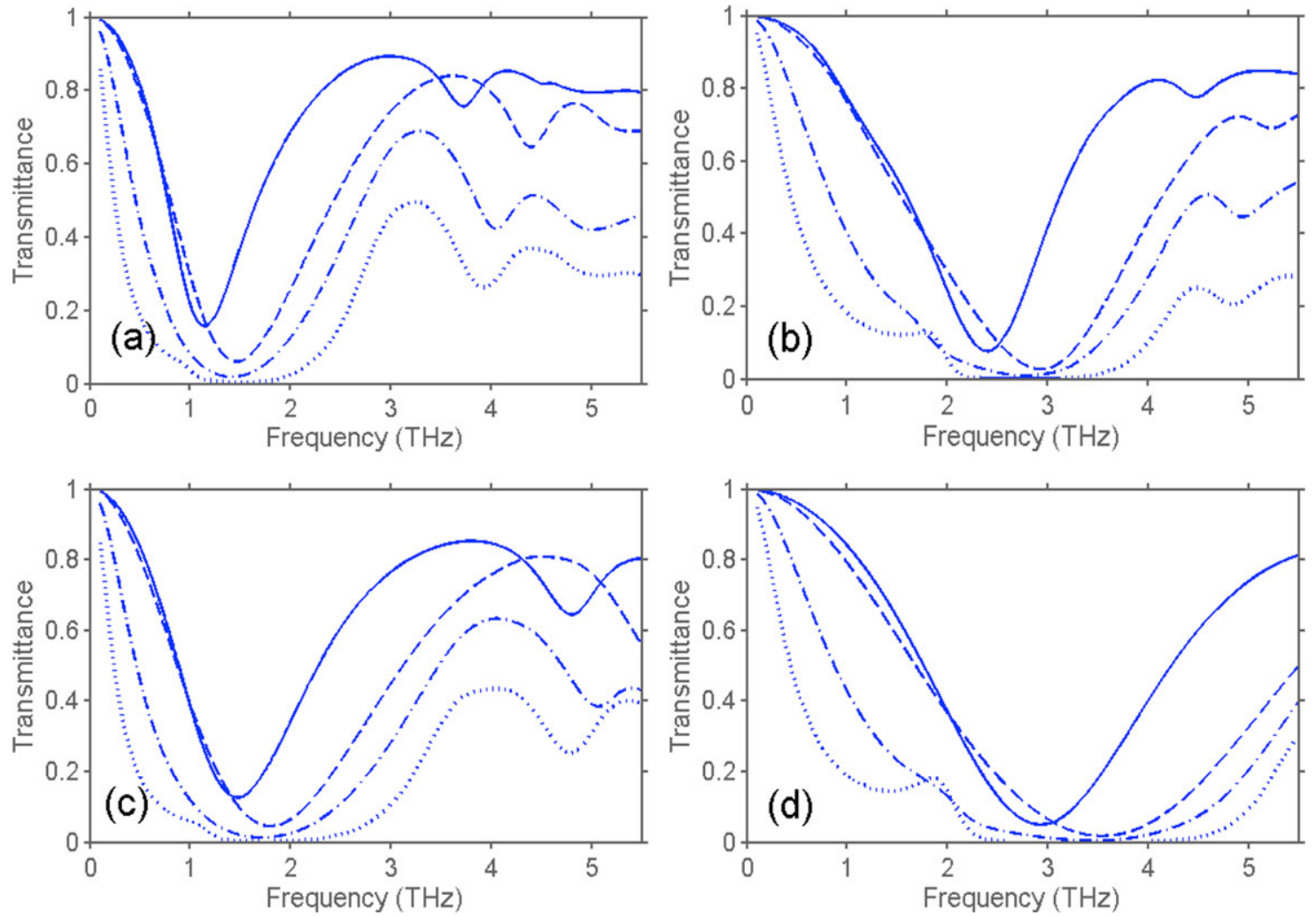

Figure 12. Computed spectra of a,c) $t_{x x}$ and $\left.\mathrm{b}, \mathrm{d}\right) t_{y y}$ for $\left.b=1 \mu \mathrm{m}, a=a_{0}, \mathrm{a}, \mathrm{b}\right) B_{0}=1 \mathrm{~T}$ in the Faraday configuration and c,d) bias-free configuration, when $\varepsilon_{d}=5.8$. Solid lines are for $M=1$, dashed lines for $M=2$, dash-dotted lines for $M=4$, and dotted lines for $M=8$.

region containing the resonance field can be affected by the substrate/spacers. The observed effects of the orientation of the magnetostatic field on the center frequencies of the stopbands include strong and (very) weak sensitivity of transmission to the changes of magnetostatic-field orientation.

We deduced approximate scaling rules of the type $f_{c} \propto \varepsilon_{d}^{-\alpha}$ from the obtained numerical results, thereby quantitatively predicting the shifts of resonances by varying $\varepsilon_{d}$. These rules expectedly differ from the classical scaling rule $f_{c} \propto \varepsilon_{d}^{-1 / 2}$ that is applicable to electromagnetic cavities, i.e., the effect of variations in $\varepsilon_{d}$ is weaker, since the substrate/spacers do not occupy the entirety of the region in which the resonance field has high magnitudes. In spite of this, variations in $\varepsilon_{d}$ remain a very efficient tool for design, because the spectral locations of subwavelength resonances still strongly depend on $\varepsilon_{d}$. In many practical cases, the effects of metasurface geometry, substrate/spacer material, and biasing field can be considered separately, at least for the purposes of initial-stage design.

Strong dependence of transmission on the polarization state of the incident wave was observed in the considered ranges of variation in the relative permittivity $\varepsilon_{d}$ of the substrate/spacers, the thickness $b$ of the substrate/spacers, and the number $M$ of cascaded metasurfaces. This dependence gives an additional and a very important degree of freedom for controlling transmission and reflection, and enables switching from one operational regime to another by means of changing the polarization state of the incident plane wave. On-off type switching can be obtained by changing the orientation of magnetostatic field, at least for one of the two mutually orthogonal linear polarizations of the incident plane wave. Thus, the mutually reinforcing effects of the variations in the polarization state of the incident plane wave and the orientation of the magnetostatic field can be exploited to enhance dynamic tunability.

The characteristic attributes delineated for $M \in\{1,2\}$ appear also at larger values of $M$. All features in the transmittance spectra for the bias-free configuration that are related to the number of cascaded metasurfaces are also observed when a magnetostatic field is applied. Thus, analysis and design can further be simplified by only keeping the degrees of freedom related to the geometry and substrate/spacer material at the first step. The obtained results give us guidelines for efficient design of practical $\mathrm{THz}$ devices which can be tuned with the aid of a magnetostatic bias field.

A full assessment of dynamic tunability would require experimental investigation of the rate at which $B_{0}$ can be varied, because the switching time is determined significantly by the response characteristics of the control circuit. Furthermore, either the theoretical solution of an initial-value boundary-value problem for the metasurface (cascade) or experimental investigation would be needed. These exercises lie outside the scope of this paper. However, the sine qua non of dynamic tunability are the sensitivities of the spectra of the response characteristics to the control parameter, which we have amply established through the solution of a boundary-value problem here. The strong sensitivity to the variations of magnitude and orientation of the magnetostatic field demonstrated in this paper indicates that 
the use of magnetically tunable resonators in metasurfaces for polarization conversion ${ }^{[23]}$ and vortex manipulation ${ }^{[13]}$ is a prospective research direction.

\section{Supporting Information}

Supporting Information is available from the Wiley Online Library or from the author.

\section{Acknowledgements}

AES has been supported by National Science Centre (NCN), Poland under the project MetaSel (DEC-2015/17/B/ST3/00118). AL thanks the Charles Godfrey Binder Endowment at the Pennsylvania State University for ongoing support of his research activities. Contribution of EO has been supported by DPT (HAMIT), TUBITAK (113E331, 114E374, 115F560), and Turkish Academy of Sciences.

\section{Conflict of Interest}

The authors declare no conflict of interest.

\section{Keywords}

magnetostatic control, metasurface cascade, terahertz filter, tunable metasurface

Received: June 28, 2017

Revised: September 19, 2017

Published online: November 15, 2017

[1] L. Zhang, S. Mei, K. Huang, C.-W. Qiu, Adv. Opt. Mat. 2016, 4, 818.

[2] A. Epstein, G. Eleftheriades, J. Opt. Soc. Am. B 2016, 33, A31.

[3] S. B. Glybovski, S. A. Tretyakov, P. A. Belov, Y. S. Kivshar, C. R. Simovski, Phys. Rep. 2016, 634, 1.

[4] C. L. Holloway, E. R. Kuester, D. R. Novotny, IEEE Antennas Wireless Propag. Lett. 2009, 8, 525.

[5] S. Walia, C. M. Shah, P. Gutruf, H. Nili, D. R. Chowdhury, W. Withayachumnankul, M. Bhaskaran, S. Sriram, Appl. Phys. Rev. 2015, 2, 011303.

[6] M. Veysi, C. Guclu, O. Boyraz, F. Capolino, J. Opt. Soc. Am. B 2015, 32, 318.

[7] J. Proust, F. Bedu, B. Gallas, I. Ozerov, N. Bonod, ACS Nano 2016, 10, 7761.

[8] J. Chen, H. Mosallaei, J. Opt. Soc. Am. B 2015, 32, 2115.

[9] M. Beruete, P. Rodriguez-Ulibarri, V. Pancheco-Pena, M. Navarro-Cia, A. E. Serebryannikov, Phys. Rev. B 2013, 87, 205128.

[10] A. E. Serebryannikov, A. Lakhtakia, E. Ozbay, J. Opt. Soc. Am. B 2016, 33, 834.

[11] R. M. Kaipurath, M. Pietrzyk, L. Caspani, T. Roger, M. Clerici, C. Rizza, A. Ciattoni, A. Di Falco, D. Faccio, Sci. Rep. 2016, 6, 27700

[12] I. Aghanejad, K. J. Chau, L. Markley, Phys. Rev. B 2016, 94, 165133.

[13] S. Mei, M. Q. Mehmood, S. Hussain, K. Huang, X. Ling, S. Y. Siew, H. Liu, J. Teng, A. Danner, C.-W. Qiu, Adv. Funct. Mater. 2016, 26, 5255.

[14] C. Pfeiffer, A. Grbic, Phys. Rev. Lett. 2013, 110, 197401.

[15] A. E. Serebryannikov, M. Mutlu, E. Ozbay, Appl. Phys. Lett. 2015, 107, 221907.

[16] R. Singh, E. Plum, C. Menzel, C. Rockstuhl, A. K. Azad, R. A. Cheville, F. Lederer, W. Zhang, N. I. Zheludev, Phys. Rev. B 2008, 80, 153104. https://doi.org/10.1103/PhysRevB.80.153104
[17] F. Qin, L. Ding, L. Zhang, F. Monticone, C. C. Chum, J. Deng, S. Mei, Y. Li, J. Teng, M. Hong, S. Zhang, A. Alu, C.-W. Qiu, Sci. Adv. 2016, 2, e 1501168 .

[18] I. B. Vendik, O. G. Vendik, M. A. Odit, D. V. Kholodnyak, S. P. Zubko, M. F. Sitnikova, P. A. Turalchuk, K. N. Zemlyakov, I. V. Munina, D. S. Kozlov, V. M. Turgaliev, A. B. Ustinov, Y. Park, J. Kihm, C.-W. Lee, IEEE Trans. Terahertz Sci. Technol. 2012, 2, 538.

[19] K. Fan, W. Padilla, Materials Today 2014, 18, 39.

[20] S. I. Sheikh, A. A. P. Gibson, B.M. Dillon, IEEE Trans. Microwave Theory Tech. 1998, 46, 62.

[21] R. Singh, A. K. Azad, Q. X. Jia, A. J. Taylor, H.-T. Chen, Opt. Lett. 2011, 36,1230

[22] J. Han, A. Lakhtakia, J. Mod. Opt. 2009, 56, 554.

[23] D. Wang, L. Zhang, Y. Gu, M. Q. Mehmood, Y. Gong, A. Srivastava, L. Jian, T. Venkatesan, C.-W. Qiu, M. Hong, Sci. Rep. 2015, 5, 15020.

[24] J. Han, A. Lakhtakia, C.-W. Qui, Opt. Express 2008, 16, 14390.

[25] M. Decker, C. Kremers, A. Minovich, I. Staude, A. E. Miroshnichenko, D. Chigrin, D. N. Neshev, C. Jagadish, Y. S. Kivshar, Opt. Express 2013, 21,8879 .

[26] H.-T. Chen, S. Palit, T. Tyler, C. M. Bingham, J. M. O. Zide, J. F. O'Hara, D. R. Smith, A. C. Gossard, R. D. Averitt, W. J. Padilla, N. M. Jokerst, A. J. Taylor, Appl. Phys. Lett. 2008, 93, 091117.

[27] J. Lee, S. Jung, P.-Y. Chen, F. Lu, F. Demmerle, G. Boehm, M.-C. Amann, A. Alu, M. A. Belkin, Adv. Opt. Mat. 2014, 2, 1057.

[28] J. Chen, M. Badioli, P. Alonso-Gonzales, S. Thongrattanasiri, F. Huth, J. Osmond, M. Spasenovic, A. Centeno, A. Pesquera, P. Godignon, A. Zurutuza Elorza, N. Camara, F. J. Garcia de Abajo, R. Hillenbrand, F. H. L. Koppens, Nature 2012, 487, 77.

[29] H.-T. Chen, W. J. Padilla, J. M. O. Zide, S. R. Bank, A. C. Gossard, A. J. Taylor, R. D. Averitt, Opt. Lett. 2007, 32, 1620.

[30] W. J. Padilla, A. J. Taylor, C. Highstrete, M. Lee, R. D. Averitt, Phys. Rev. Lett. 2006, 96, 107401.

[31] T. G. Mackay, A. Lakhtakia, Phys. Rev. A 2015, 92, 053847.

[32] A. E. Serebryannikov, A. Lakhtakia, Opt. Lett. 2013, 38, 3279.

[33] J. Han, A. Lakhtakia, Z. Tian, X. Lu, W. Zhang, Opt. Lett. 2009, 34, 1465.

[34] T. F. Gundogdu, M. Gokkavas, K. Guven, M. Kafesaki, C. M. Soukoulis, E. Ozbay, Photon. Nanostr. Fundam. Appl. 2007, 5, 106.

[35] N.-H. Shen, M. Massaouti, M. Gokkavas, J.-M.Manceau, E. Ozbay, M. Kafesaki, T. Koschny, S. Tzortzakis, C. M. Soukoulis, Phys. Rev. Lett. 2011, 106, 037403

[36] A. Lakhtakia, D. E. Wolfe, M. W. Horn, J. Mazurowski, A. Burger, P. P. Banerjee, Proc. SPIE 2017, 10162, 101620V.

[37] J. Hao, Y. Yuan, L. Ran, T. Jiang, C.T. Chan, L. Zhou, Phys. Rev. Lett. 2007, 99, 063908.

[38] S. Campione, J. R. Wendt, G. A. Kleer, T. S. Luk, ACS Photon. 2016, 3 , 293.

[39] J. A. Harrington, Time-Harmonic Electromagnetic Fields, McGraw-Hill, New York 1968.

[40] See www.cst.com for software details.

[41] T. Q. Li, H. Liu, T. Li, S. M. Wang, F. M. Wang, R. X. Wu, P. Chen, S. N. Zhu, X. Zhang, Appl.Phys. Lett. 2008, 92, 131111.

[42] H. Liu, J. X. Cao, S. N. Zhu, N. Liu, R. Ameling, H. Giessen, Phys. Rev. B 2010, 81, 241403.

[43] H. Liu, D. A. Genov, D. M. Wu, Y. M. Liu, Z. W. Liu, C. Sun, S. N. Zhu, X. Zhang, Phys. Rev. B 2010, 76, 073101.

[44] N. Liu, H. Liu, S. Zhu, H. Giessen, Nat. Photon. 2009, 3, 157.

[45] V. A. Yakubovich, V. M. Starzhinskii, Linear Differential Equations with Periodic Coefficients, Wiley, New York 1975.

[46] G. A. Sinclair, Proc. IRE 1948, 36, 1364

[47] A. Lakhtakia, Microwave Opt. Technol. Lett. 1994, 7, 328. 
[48] T. Driscoll, G. O. Andreev, D. N. Basov, S. Palit, S. Y. Cho, N. M. Jokerst, D. R. Smith, Appl. Phys. Lett. 2007, 91, 062511.

[49] S.-Y. Chiam, R. Singh, J. Gu, J. Han, W. Zhang, A. Bettiol, Appl. Phys. Lett. 2009, 94, 064102.

[50] X. Gan, R.-J. Shiue, Y. Gao, S. Assefa, J. Hone, D. Englund, IEEE Trans. Select. Top. Quant. Electron. 2014, 20,6000311.

[51] K. Song, Y. Liu, Q. Fu, X. Zhao, C. Luo, W. Zhu, Opt. Express 2013, 21, 7439.

[52] K. Song, X. Zhao, Y. Li, Q. Fu, C. Luo, Appl. Phys. Lett. 2013, 103, 101908.
[53] Y. Yao, M.A. Kats, P. Shankar, Y. Song, J. Kong, M. Loncar, F. Capasso, Nano Lett. 2014, 14, 214.

[54] X. Xia, Y. Sun, H. Yang, H. Feng, L. Wang, C. Gua, J. Appl. Phys. 2008, 104, 033505.

[55] M. W. Klein, C. Enkrich, M. Wegener, C. M. Soukoulis, S. Linden, Opt. Lett. 2006, 31, 1259.

[56] T. Zentgraf, T. P. Meyrath, A. Seidel, S. Kaiser, H. Giessen, Phys. Rev. B 2007, 76, 033407.

[57] R. A. Waldron, Proc. IEE 1960, 107C, 272.

[58] A. Lakhtakia, Int. J. Infra. Millimeter Waves 1991, 12, 109. 